\title{
Thermally Driven Tropical Circulations under Rayleigh Friction and Newtonian Cooling: Analytic Solutions*
}

\author{
Zhaohua Wu, E. S. Sarachik, and David S. Battisti \\ Department of Atmospheric Sciences, University of Washington, Seattle, Washington
}

(Manuscript received 13 December 1999, in final form 20 July 2000)

\begin{abstract}
In this paper, the atmospheric circulations on an equatorial beta plane in response to steady tropical heating are investigated by analytically solving a set of linear equations. Special emphasis is placed on the horizontal structure of forced response under the different combinations of momentum damping and thermal damping, as well as the effect of the zonal domain on the forced responses. Two zonal domains are considered: a zonally cyclic domain and a zonally unbounded domain.

The linear model is decomposed in terms of the vertical eigenfunctions in a vertically semi-infinite domain. A new feature of the solution is the existence of a continuous spectrum corresponding to energy propagation out the top of the troposphere. The resulting shallow-water equations are then solved using a method similar to that of Gill.

Since the zonal decay scale is proportional to the inverse of the square root of the product of the Rayleigh friction rate and the Newtonian cooling rate, the solutions in a zonally unbounded domain can be good approximations for the solutions in a zonally cyclic domain only when both Rayleigh friction and Newtonian cooling are large enough. When either Rayleigh friction or Newtonian cooling is very weak, the solutions are essentially zonally uniform regardless of the longitudinal location of the heat source in a zonally cyclic domain except in a very narrow zone along the equator.

The characteristic meridional scale of the shallow-water system is the equatorial radius of deformation of the shallow-water system multiplied by the fourth root of the ratio between the Rayleigh friction rate and the Newtonian cooling rate. Therefore, the characteristic meridional scale is very large for the Rayleigh frictiondominant case, and the forced response can extend far outside the heating latitude. In contrast, in the Newtonian cooling-dominant case the characteristic meridional scale is very small and the forced response is confined to the heating latitudes.

The implications of these solutions for both the thermally driven surface winds and the zonally uniform lowfrequency variation in pressure and temperature in the upper half of the tropical troposphere are also discussed.
\end{abstract}

\section{Introduction}

Rayleigh friction and Newtonian cooling are simple idealizations of physical processes in the atmosphere and ocean, such as nonlinear effects (Holton and Colton 1972), eddy viscosity, and eddy diffusivity (McCreary 1981). The ratio between Rayleigh friction and Newtonian cooling, called the Prandtl number (Pr), is an important quantity comparing the relative importance of momentum damping and thermal damping.

The roles of thermal damping and momentum damping in the tropical atmosphere have been studied for a

\footnotetext{
* Joint Institute for the Study of the Atmosphere and Ocean Contribution Number 686 .

Corresponding author address: Dr. Zhaohua Wu, Center for Ocean-Land-Atmosphere Studies, 4041 Powder Mill Road, Suite 302, Calverton, MD 20705-3106.

E-mail: zhwu@cola.iges.org
}

long time. Lindzen (1968) investigated the behavior of vertically propagating waves in an atmosphere with small Newtonian cooling rate near the ground, and increasing inversely with atmospheric density away from the ground. His results demonstrated that the amplitude of the divergence $(\partial u / \partial x+\partial v / \partial y)$ does not vary with height at high altitudes where Newtonian cooling becomes very strong. Dickinson and Geller (1968) further showed that the method used in Lindzen (1968) can be applied to the tidal problem using Newtonian cooling with a more complex vertical structure. Shapiro (1977) examined the importance of boundary layer friction for thermally forced gravity and Rossby waves. Chang (1977) explored thermally driven low-frequency oscillations in the Tropics in the presence of both Rayleigh friction and Newtonian cooling (both having the same damping timescale). He demonstrated that inclusion of simple linear damping has a strong influence at low frequencies on the forced equatorial waves, resulting in a different dispersion relationship from that without dissipation. Haarsma and Opsteegh (1989), through their 

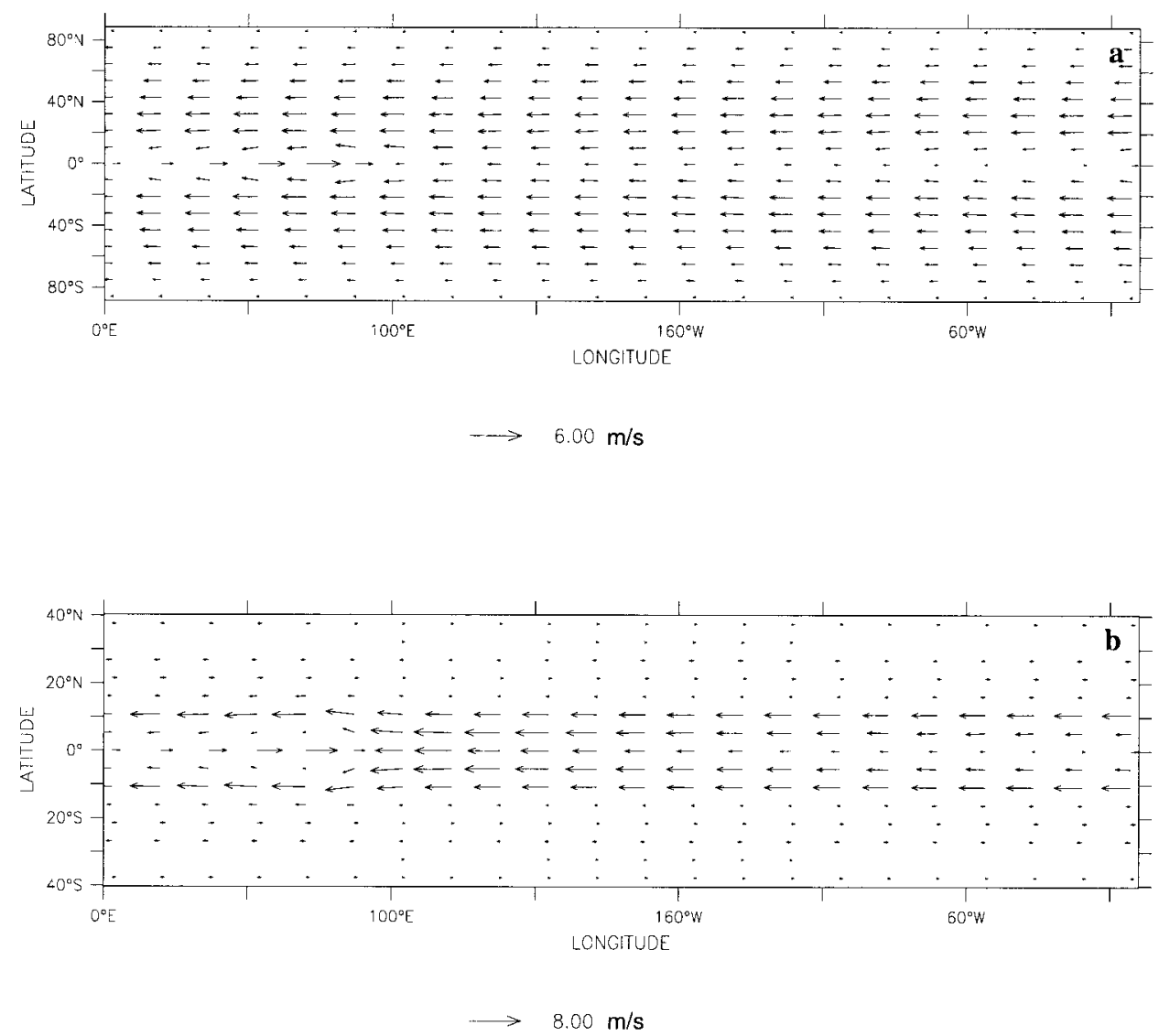

FIG. 1. The horizontal wind fields at $760 \mathrm{hPa}$ for (a) the Rayleigh friction-alone case and (b) the Newtonian cooling-alone case in a dry primitive equation general circulation model. The scale of the horizontal velocity is shown under each panel. The prescribed heating is given in appendix A with its vertical structure being illustrated by the solid line in Fig. 2b. The heat source of Gaussian shape with zonal scale of $20^{\circ}$ and meridional scale of $11^{\circ}$ is applied centered at the equator and $93^{\circ} \mathrm{E}$.

study of the circulations driven by thermal sources in the Tropics, showed that Rayleigh friction affects the midlatitude flow patterns in response to the tropical heating, and affects the transition threshold from linear to nonlinear regime. Kato (1997) used a global linear shallow-water model to show that the flow patterns driven by a propagating solar insolation-like heat source are sensitively dependent on Rayleigh friction rather than Newtonian cooling. Wu et al. (1999), by examining the projection of large-scale heating onto both the discrete modes and continuous modes and the corresponding vertical structure equations for these modes, found that the Newtonian cooling can dramatically change the vertical structure of the forced response and bring strong forced signal to the surface. Plumb and Eluszkiewicz (1999) showed the importance of the effects of weak model viscosity (some 50 times weaker than thermal relaxation) in permitting flow across angular momentum contours within a tropical boundary layer and explored the possibility that diabatic heating alone can provide the tropical upwelling for a Brewer-Dobson circulation in the stratosphere.
The influence of eddy viscosity on oceanic waves in the Tropics has also been extensively explored. Martinsen and Weber (1981), through a decomposition of variables in terms of the vertical normal modes, examined the influence of eddy viscosity on internal Kelvin waves; Mofjeld (1981), using a shallow-water model, investigated free internal wave solutions in the presence of linear (Rayleigh) friction in the equatorial waveguide. The latter study demonstrated that at low frequencies 1) friction makes the meridional scale of the equatorial waveguide significantly larger than the inviscid scale, and 2) there is relatively little zonal damping of Kelvin, inertial-gravity, and Rossby waves.

One study that systematically explored the roles of Rayleigh friction and Newtonian cooling in the Tropics was Yamagata and Philander (1985). They showed that, for the steady shallow-water system, the original coordinates $(x, y)$ can be transformed into new coordinates $(X, Y)\left[x=(\alpha / \gamma)^{1 / 2} X, y=(\alpha / \gamma)^{1 / 4} Y\right]$, with appropriate scalings on the independent variables, such that the original shallow-water system with differing values for the Rayleigh friction rate and the Newtonian cooling rate 

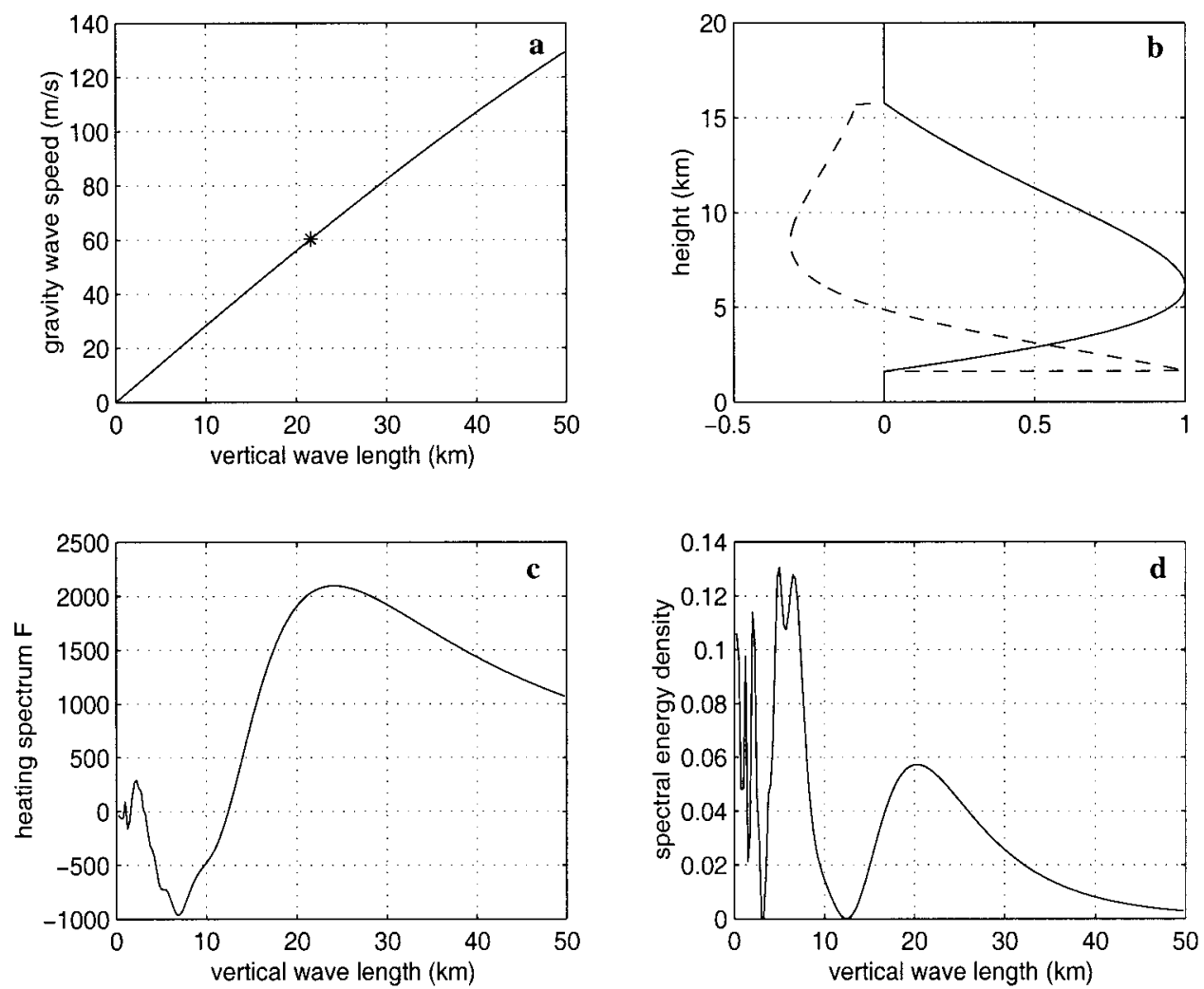

FIG. 2. (a) The plot of the gravity wave speed against the vertical wave length for a baroclinic mode in an isothermal atmosphere of $300 \mathrm{~K}$. The "**" point corresponds to the gravity speed of $60 \mathrm{~m} \mathrm{~s}^{-1}$. (b) The normalized vertical heating profiles used in both the numerical modeling study and the analytical studies. The solid line represents the vertical structure of the external heating given by Eq. (A.2), and the dashed line represents the corresponding vertical structure of $\tilde{Q}$ in Eq. (2.10). (c) The projection [the spectrum $F(l)$ ] of $P(z)$ onto the vertical eigenfunctions. (d) The spectral energy density of the heating with respect to vertical wavelength $l$. See section 2 for the definitions of $F(l), P(z)$, and spectral energy density.

$(\operatorname{Pr} \neq 1)$ can be transformed into the standard shallowwater system with identical values for the Rayleigh friction rate and the Newtonian cooling rate $(\mathrm{Pr}=1)$ in the new coordinates $(X, Y)$. From these results, they inferred that the zonal damping scale for the Kelvin signal in the original coordinates is characterized by the inverse of $\sqrt{\alpha \gamma}$, where $\alpha$ is the Rayleigh friction rate and $\gamma$ is the Newtonian cooling rate. They also performed numerical studies using a nonlinear shallowwater model for different combinations of the Rayleigh friction rate and the Newtonian cooling rate in a rectangle domain with rigid lateral boundaries. Unfortunately, since their focus was on the oceanic response to basinwide wind stress, and since they could not eliminate the effect of the strong coastal Kelvin waves in their numerical experiments, their results cannot be easily applied to the atmosphere forced by an isolated thermal source in the Tropics. Yamagata and Hayashi (1984), to study intraseasonal oscillations in the Tropics, carried out some calculations for the circulations driven by an isolated heat source in the tropics using different values of the Rayleigh friction rate and the Newtonian cooling rate. Later, Clarke (1994) used similar scaling method to calculate the heat-induced low-level circulations and presented an explanation for why surface equatorial anomalous westerlies occur under anomalous large-scale convection on interannual timescales.

Recently, Wu et al. (2000a) studied the different roles of Rayleigh friction and Newtonian cooling in a thermally forced tropical atmosphere, both numerically using a dry primitive equation GCM, and semianalytically solving simple linear models. Their results showed that dominant Newtonian cooling homogenizes the atmospheric motion in the vertical direction, and a strong, vertically uniform wind is found below the bottom of the heat source. When Rayleigh friction dominates, the circulations driven by the heat source are confined to the layer where the heat source is located. These results provide the clues to understand thermally driven surface winds in the Tropics that are crucial to the coupling between atmosphere and ocean. Wu et al. (2000b) further investigated how vertical structure of heating affects the forced circulation and found that the vertical distribuation of heating is one of the key factors that determine the three-dimensional structures of the forced circulations. 
A feature not fully explained in Wu et al. (2000a) is the horizontal structures of the flow driven by an isolated heat source (see appendix A and Fig. 2b for details) in different damping cases, as shown by Fig. 1. In both the Rayleigh friction-alone case and the Newtonian coolingalone case, the forced flows in the lower half of the troposphere are almost everywhere easterlies that are quite zonally uniform except a very narrow zone along the equator. In the Newtonian cooling-alone case, the thermally driven flow is confined to the heating latitudes, while in the Rayleigh friction-alone case, the flow resembles a Gaussian shape in the meridional direction with a scale of about $60^{\circ}$ except in a meridionally very narrow zone along the equator. These results cannot be explained by the classical Gill model with a zonally infinite domain that predicts Kelvin signal to the east of the heating and Rossby signals to the west of the heating. Hence, it is of theoretical and practical significance to understand how Rayleigh friction and Newtonian cooling play their contrasting roles in the tropical atmosphere.

In this study, we present an analytical linear theory of the thermally forced tropical atmosphere. This study can be regarded as an theoretical extension of $\mathrm{Wu}$ et al. (2000a). Since the vertical decomposition method have already been developed in detail in Wu et al. (2000b), the focus of this paper will be on the steady response of the shallow-water system to a prescribed local heating under different combinations of Rayleigh friction and Newtonain cooling. In section 2 , the governing equations, the vertical eigenfunctions for a semi-infinite atmosphere, and the vertical decomposition method will be outlined, and the spectral distribution of the heating (in terms of the vertical eigenfunctions) will be calculated. In section 3, an analytical method for solving a locally forced shallow-water system in a zonally cyclic domain is developed. Calculations of the circulation driven by the heating with the same horizontal structure but using different dampings will be carried out in section 4. The implications of the present study to the thermally driven surface winds and to the zonally uniform low-frequency variation in pressure and temperature in the Tropics will be discussed in section 5. A summary will be presented in section 6 .

\section{Basic equations}

The linear equations in log pressure coordinates for the tropical atmosphere on an equatorial $\beta$ plane are

$$
\begin{aligned}
\left(\frac{\partial}{\partial t}+\alpha\right) u^{*}-\beta y v^{*} & =-\frac{\partial \Phi^{*}}{\partial x}, \\
\left(\frac{\partial}{\partial t}+\alpha\right) v^{*}+\beta y u^{*} & =-\frac{\partial \Phi^{*}}{\partial y}, \\
\frac{\partial u^{*}}{\partial x}+\frac{\partial v^{*}}{\partial y}+\frac{1}{\rho_{0}} \frac{\partial\left(\rho_{0} w^{*}\right)}{\partial z^{*}} & =0, \quad \text { and } \\
\left(\frac{\partial}{\partial t}+\gamma\right)\left(\frac{\partial \Phi^{*}}{\partial z^{*}}\right)+N^{2} w^{*} & =\frac{\kappa J}{H},
\end{aligned}
$$

where $u^{*}\left(v^{*}\right)$ is the eastward (northward) velocity, $w^{*}$ is the vertical velocity in log pressure coordinates, $\Phi^{*}$ is the geopotential, $\rho_{0}$ is the basic density, $\kappa$ is the gas constant $R$ divided by the heat capacity $c_{p}, J$ is the external heating rate per unit mass per unit time, $N^{2}$ is the buoyancy frequency squared, and $H$ is a scale height. Here, $\beta=2 \Omega / a$, and $\Omega$ and $a$ are the earth's rotation rate and radius, respectively; $\alpha$ is Rayleigh friction coefficient; and $\gamma$ is Newtonian cooling coefficient.

Equations (2.1) use an equatorial beta-plane approximation to the sphere and are standard for studying the tropical atmosphere (see, e.g., Holton 1992). Although the generalization to a nonisothermal atmosphere is straightforward, we simplify the algebra by assuming an isothermal atmosphere of temperature $T_{s}$. The basic state is taken to be motionless. Hence, the basic density $\rho_{0}$ satisfies

$$
\rho_{0}\left(z^{*}\right)=\rho_{0}(0) e^{-z * / H} .
$$

To simplify Eqs. (2.1), we introduce the following mass-weighted variables: $u=\rho_{0}^{1 / 2} u^{*}, v=\rho_{0}^{1 / 2} v^{*}, w=$ $\rho_{0}^{1 / 2} w^{*}$, and $\Phi=\rho_{0}^{1 / 2} \Phi^{*}$. These are substituted into Eqs. (2.1) using Eq. (2.2), and time dependence of the form $e^{-i \omega t}$ is assumed to obtain

$$
\begin{aligned}
(\alpha-i \omega) u-\beta y v & =-\frac{\partial \Phi}{\partial x}, \\
(\alpha-i \omega) v+\beta y u & =-\frac{\partial \Phi}{\partial y}, \\
\frac{\partial u}{\partial x}+\frac{\partial v}{\partial y}+\left(\frac{\partial}{\partial z}-\frac{1}{2 H}\right) w & =0, \quad \text { and } \\
(\gamma-i \omega)\left(\frac{\partial}{\partial z}+\frac{1}{2 H}\right) \Phi+w N^{2} & =Q,
\end{aligned}
$$

where the superscript " $*$ " has been omitted from $z$ and $Q=\rho_{0}^{1 / 2} \kappa J / H$.

Eliminating $w$ from Eqs. (2.3c) and (2.3d) leads to

$$
\begin{gathered}
\frac{\gamma-i \omega}{N^{2}}\left(\frac{\partial^{2}}{\partial z^{2}}-\frac{1}{4 H^{2}}\right) \Phi+-\left(\frac{\partial u}{\partial x}+\frac{\partial v}{\partial y}\right) \\
=\left(\frac{\partial}{\partial z}-\frac{1}{2 H}\right) \frac{Q}{N^{2}} .
\end{gathered}
$$

The vertical eigenfunctions are defined by the equation.

$$
\frac{d^{2} V_{m}}{d z^{2}}+\left(\frac{N^{2}}{g h_{m}}-\frac{1}{4 H^{2}}\right) V_{m}=0
$$

and the boundary conditions

$$
\begin{aligned}
& \frac{d V_{m}}{d z}+\frac{V_{m}}{2 H}=0, \quad \text { at } z=0, \text { and } \\
& \text { either } V_{m} \rightarrow 0 \text { or }
\end{aligned}
$$

outgoing radiation condition, as $a \rightarrow \infty$. 
Equation (2.5) and its boundary conditions [Eqs. (2.6a) and (2.6b)] define a complete set of eigenfunctions in the vertical under the concept of analytical continuation (Friedman 1956). From Wu et al. (2000b), we know that these eigenfunctions include a discrete mode (often called the external mode or the barotropic mode)

$$
V_{e}(z)=e^{-z / 2 H},
$$

and the continuous modes (often called the internal modes or the baroclinic modes)

$V_{m}(z)=\left(\cos m z-\frac{1}{2 H m} \sin m z\right) \frac{2 H m}{\sqrt{4 H^{2} m^{2}+1}}$,

with $m$ being any real number.

Although the vertical profile of an eigenfunction with a given vertical wave number is fixed, the physical meaning of the eigenfunctions expressed by Eq. (2.7b) is different from that of the traditional "normal modes" that result from imposing an artificial lid at the top of the model atmosphere. An artificial lid prevents energy from propagating out to space, generates many dominant resonant structures (normal modes) in the vertical, and allows the existence of free solutions. However, the eigenfunctions expressed by Eq. (2.7b) imply radiation of energy to space [see Wu et al. (2000b) for details]. Therefore, there exists no free solution other than the solution (2.7a), which is uniform in the vertical, in a vertically semi-infinite atmosphere, and only forced waves can survive.

By expanding the variables in Eqs. (2.3a), (2.3b), and (2.4) in terms of the vertical eigenfunctions $V_{m}$, that is,

$$
\left[\begin{array}{c}
u \\
v \\
\Phi
\end{array}\right]=\left[\begin{array}{c}
u_{e}(x, y) \\
v_{e}(x, y) \\
\Phi_{e}(x, y)
\end{array}\right] e^{-(z / 2 H)}+\int_{0}^{\infty}\left[\begin{array}{c}
u_{m}(x, y) \\
v_{m}(x, y) \\
\Phi_{m}(x, y)
\end{array}\right]\left[\left(\cos m z-\frac{1}{2 H m} \sin m z\right) \frac{2 H m}{\sqrt{4 H^{2} m^{2}+1}}\right] d m,
$$

we obtain

$$
\begin{aligned}
& (\alpha-i \omega) u_{s}-\beta y v_{s}=-\frac{\partial \Phi_{s}}{\partial x} \\
& (\alpha-i \omega) v_{s}-\beta y u_{s}=-\frac{\partial \Phi_{s}}{\partial y}, \quad \text { and }
\end{aligned}
$$

$$
\frac{(\gamma-i \omega)}{g h_{s}} \Phi_{s}+\left(\frac{\partial u_{s}}{\partial x}+\frac{\partial v_{s}}{\partial y}\right)=-F_{s}
$$

where the subscript $s$ represents either $e$ or $m$ and $F_{s}(x, y)$ satisfies

$$
\tilde{Q}=\left(\frac{\partial}{\partial z}-\frac{1}{2 H}\right) \frac{Q}{N^{2}}=F_{e} e^{-(z / 2 H)}+\int_{0}^{\infty} F_{m}\left[\left(\cos m z-\frac{1}{2 H m} \sin m z\right) \frac{2 H m}{\sqrt{4 H^{2} m^{2}+1}}\right] d m
$$

Equations (2.9) are the shallow-water equations for a homogeneous fluid of a depth $h_{s}$. These equations are combined to form a single equation for the meridional velocity $v_{s}$ :

$$
\begin{aligned}
& {\left[\frac{\partial^{2}}{\partial y^{2}}+\frac{\partial^{2}}{\partial x^{2}}+\frac{\beta}{(\alpha-i \omega)} \frac{\partial}{\partial x}\right] v_{s}} \\
& -\frac{\gamma-i \omega}{g h_{s}(\alpha-i \omega)}\left[\beta^{2} y^{2}+(\alpha-i \omega)^{2}\right] v_{s}=\tilde{F}_{s},
\end{aligned}
$$

where

$$
\tilde{F}_{s}=\frac{1}{\alpha-i \omega}\left[\beta y \frac{\partial}{\partial x}-(\alpha-i \omega) \frac{\partial}{\partial y}\right] F_{s} .
$$

For simplicity, we assume that the heating rate $\tilde{Q}$ in Eq. (2.10) has the form $P(z) S(x, y)$. Then, we obtain

$$
F_{s}(x, y)=F(s) S(x, y),
$$

where $F(s)$ is the projection of $P(z)$ onto the vertical eigenfunction $V_{s}(z)$. The expression of $P(z)$ in terms of the vertical eigenfunctions is

$$
\begin{aligned}
& P(z)= F_{e} e^{-(z / 2 H)} \\
&+\sqrt{\frac{2}{\pi}} \int_{0}^{\infty} F_{m}(m)\left(\cos m z-\frac{1}{2 H m} \sin m z\right) \\
& \quad \times \frac{2 H m}{\sqrt{4 H^{2} m^{2}+1}} d m
\end{aligned}
$$

where $F_{e}$ is the projection of $P(z)$ onto the external mode 


$$
F_{e}=\frac{1}{H} \int_{0}^{\infty} P(z) e^{-(z / 2 H)} d z,
$$

and $F_{m}(m)$ is the projection of $P(z)$ onto an internal mode

$$
\begin{gathered}
F_{m}(m)=\sqrt{\frac{2}{\pi} \int_{0}^{\infty}} P(z)\left(\cos m z-\frac{1}{2 H m} \sin m z\right) \\
\times \frac{2 H m}{\sqrt{4 H^{2} m^{2}+1}} d z .
\end{gathered}
$$

If we consider the heat source as the parameterization of the large-scale convective heating, generally its projection onto the barotropic mode is small. Hence, the Parseval's theorem can be written as

$$
\begin{aligned}
E & =\int_{0}^{\infty}|P(z)|^{2} d z \approx \int_{0}^{\infty} F_{m}^{2}(m) d m \\
& =\int_{0}^{\infty} \frac{2 \pi F^{2}\left(l_{m}\right)}{l_{m}^{2}} d l_{m},
\end{aligned}
$$

where $l_{m}=2 \pi / m$. It is clear that $F_{m}^{2}(m)$ is the power spectrum and $2 \pi F_{m}^{2}\left(l_{m}\right) / l_{m}^{2}$ represents the spectral energy density as a function of $l_{m}$. Hence, the relative importance of the different modes in accounting for the overall response can then be quantified by these two functions. Figures $2 \mathrm{c}$ and $2 \mathrm{~d}$ are the spectrum and spectral energy density for the heat source described in appendix A and plotted in Fig. 2b. Figure 2a gives the relation between the vertical wavelength of a mode and its corresponding horizontal gravity wave speed for an isothermal atmosphere of $300 \mathrm{~K}$. The distributions of the spectrum and spectral energy density of the heat source show that the modes with the gravity wave speed $c_{m}\left(=\sqrt{g h_{m}}\right.$ with $h_{m}$ the equivalent depth) around 20 and $60 \mathrm{~m} \mathrm{~s}^{-1}$ are very important.

\section{Steady responses to an isolated heat source}

It is convenient to convert the shallow-water Eqs. (2.9) into a nondimensional form. The units of time, length, geopotential, and forcing are as follows:

$$
\begin{aligned}
& {[T]=\left(\beta \sqrt{g h_{m}}\right)^{-1 / 2}, \quad[L]=\left(\sqrt{g h_{m}} / \beta\right)^{1 / 2},} \\
& {[\Phi]=[L]^{2} /[T]^{2} \text {, and }[F]=[L]^{2} /[T]^{3} \text {, }}
\end{aligned}
$$

where $\left(\sqrt{g h_{m}} / \beta\right)^{1 / 2}$ is the equatorial radius of deformation, an intrinsic scale of the shallow-water system. Using these units, one obtains the nondimensional form of the frictional shallow-water equations for the steady case:

$$
\begin{aligned}
\alpha u-y v & =-\frac{\partial \Phi}{\partial x} \\
\alpha v-y u & =-\frac{\partial \Phi}{\partial y} \\
\gamma \Phi+\left(\frac{\partial u}{\partial x}+\frac{\partial v}{\partial y}\right) & =-F .
\end{aligned}
$$

All the variables in Eqs. (3.1a-c) are defined in a zonally cyclic domain. In the meridional direction, the boundary conditions are assumed to be $u, v, \Phi \rightarrow 0$ at $y \rightarrow \pm \infty$. Combining of Eqs. (3.1a-c) leads to a single equation of variable $v$ :

$$
\begin{gathered}
{\left[\frac{\partial^{2}}{\partial y^{2}}+\frac{\partial^{2}}{\partial x^{2}}+\frac{1}{\alpha} \frac{\partial}{\partial x}\right] v-\frac{\gamma}{\alpha}\left[y^{2}+\alpha^{2}\right] v} \\
=\frac{1}{\alpha}\left[y \frac{\partial}{\partial x}-\alpha \frac{\partial}{\partial y}\right] F .
\end{gathered}
$$

Equation (3.2) can be solved by using the variable separation method. The corresponding meridional eigenfunction $\psi_{n}(y)$ has the form

$$
\psi_{n}(y)=\exp \left(-\frac{y^{2}}{2 R^{2}}\right) H_{n}\left(\frac{y}{R}\right),
$$

which satisfies the differential equation

$$
\frac{d^{2} \psi_{n}}{d y^{2}}+\left[\frac{(2 n+1)}{R^{2}}-\frac{y^{2}}{R^{4}}\right] \psi_{n}=0,
$$

where $R=(\alpha / \gamma)^{1 / 4}$ is called the nondimensional effective equatorial radius of deformation of the frictional shallow-water system, $H_{n}$ are class of Hermite polynomials, and $n$ is a nonnegative integer. The corresponding effective equatorial radius of deformation of the shallow-water system with different damping rates of Rayleigh friction and Newtonian cooling $(\operatorname{Pr} \neq 1)$ in dimensional form is therefore the equatorial radius of deformation multiplied by the fourth root of the Prandtl number, that is, $(\alpha / \gamma)^{1 / 4}\left(\sqrt{g h_{m}} / \beta\right)^{1 / 2}$.

The set $\left\{\psi_{n}(y)\right\}$ is complete and orthogonal, with recursion relations

$$
\begin{aligned}
& R^{2} \frac{d \psi_{n}}{d y}+y \psi_{n}=2 n R \psi_{n-1}, \quad \text { and } \\
& R^{2} \frac{d \psi_{n}}{d y}-y \psi_{n}=-R \psi_{n+1} .
\end{aligned}
$$

To simplify the mathematics, we assume a semigeostrophic balance, with the eastward flow balancing the pressure gradient. As pointed out by Gill (1980), this approximation is equivalent to making a "longwave" approximation in the transient problem. Thus, Eq. (3.1b) becomes

$$
y u=-\frac{\partial \Phi}{\partial y} .
$$

Following Gill (1980), we also introduce two new variables:

$$
\begin{aligned}
& q=\sqrt{\alpha} u+\sqrt{\gamma} \Phi \text { and } \\
& r=\sqrt{\gamma} \Phi-\sqrt{\alpha} u .
\end{aligned}
$$

Combining Eqs. (3.1a) and (3.1c) leads to the equations for the new variables: 


$$
\sqrt{\alpha} q+\frac{1}{\sqrt{\gamma}} \frac{\partial q}{\partial x}-y v+\sqrt{\frac{\alpha}{\gamma}} \frac{\partial v}{\partial y}=-\sqrt{\frac{\alpha}{\gamma}} F
$$

and

$$
\sqrt{\alpha} r-\frac{1}{\sqrt{\gamma}} \frac{\partial r}{\partial x}+y v+\sqrt{\frac{\alpha}{\gamma}} \frac{\partial v}{\partial y}=-\sqrt{\frac{\alpha}{\gamma}} F,
$$

while Eq. (3.6) can be written as

$$
y q+\sqrt{\frac{\alpha}{\gamma}} \frac{\partial q}{\partial y}-y r+\sqrt{\frac{\alpha}{\gamma}} \frac{\partial r}{\partial y}=0 .
$$

Expanding all $q, r, v$, and $F$ in terms of the meridional eigenfunctions $\psi_{n}(y)$, that is,

$$
\left[\begin{array}{l}
q \\
r \\
v \\
F
\end{array}\right]=\sum_{n}\left[\begin{array}{l}
q_{n}(x) \\
r_{n}(x) \\
v_{n}(x) \\
f_{n}(x)
\end{array}\right] \psi_{n}(y),
$$

and substituting Eqs. (3.9) into Eqs. (3.8a-c), one obtains [cf. Gill (1980) for the case in which the Rayleigh friction rate and the Newtonian cooling rate are identical]

$$
\begin{gathered}
\sqrt{\alpha} \sum_{n} q_{n} \psi_{n}+\frac{1}{\sqrt{\gamma}} \sum_{n} \frac{d q_{n}}{d x} \psi_{n}-y \sum_{n} v_{n} \psi_{n}+\sqrt{\frac{\alpha}{\gamma}} \sum_{n} v_{n} \frac{d \psi_{n}}{d y}=-\sqrt{\frac{\alpha}{\gamma}} \sum_{n} f_{n} \psi_{n}, \\
\sqrt{\alpha} \sum_{n} r_{n} \psi_{n}-\frac{1}{\sqrt{\gamma}} \sum_{n} \frac{d r_{n}}{d x} \psi_{n}+y \sum_{n} v_{n} \psi_{n}+\sqrt{\frac{\alpha}{\gamma}} \sum_{n} v_{n} \frac{d \psi_{n}}{d y}=-\sqrt{\frac{\alpha}{\gamma}} \sum_{n} f_{n} \psi_{n}, \quad \text { and } \\
y \sum_{n} q_{n} \psi_{n}+\sqrt{\frac{\alpha}{\gamma}} \sum_{n} q_{n} \frac{d \psi_{n}}{d y}-y \sum_{n} r_{n} \psi_{n}+\sqrt{\frac{\alpha}{\gamma}} \sum_{n} r_{n} \frac{d \psi_{n}}{d y}=0,
\end{gathered}
$$

respectively. Using the recursive relationships $(3.5 \mathrm{a}, \mathrm{b})$ and the orthogonality property of $\psi_{n}(y)$, one obtains

$$
\begin{aligned}
& \frac{d q_{n}}{d x}+\sqrt{\alpha \gamma} q_{n}=-\sqrt{\alpha} f_{n}+(\alpha \gamma)^{1 / 4} v_{n-1}, \\
& \frac{d r_{n}}{d x}-\sqrt{\alpha \gamma} r_{n}=\sqrt{\alpha} f_{n}+2(n+1)(\alpha \gamma)^{1 / 4} v_{n+1},
\end{aligned}
$$

and

$$
2(n+1) q_{n+1}-r_{n-1}=0 .
$$

After manipulating Eqs. (3.11a-c), one obtains

$$
\begin{gathered}
\frac{d q_{0}}{d x}+\sqrt{\alpha \gamma} q_{0}=-\sqrt{\alpha} f_{0}, \\
q_{1}=0 \\
\frac{d q_{n+1}}{d x}-(2 n+1) \sqrt{\alpha \gamma} q_{n+1} \\
=\frac{\sqrt{\alpha}}{2}\left(f_{n-1}+2 n f_{n+1}\right) \quad \text { for } n \geq 1, \\
(\alpha \gamma)^{1 / 4} v_{0}=\sqrt{\alpha} f_{1}, \\
\left.+\frac{\sqrt{\alpha}}{2} f_{n-1}^{1 / 2}\right) v_{n}=2(n+1) \sqrt{\alpha \gamma} q_{n+1}+(n+1) \sqrt{\alpha} f_{n+1} \\
r_{n-1}=2(n+1) q_{n+1} \quad \text { for } n \geq 1
\end{gathered}
$$

Thus, only the variables $q_{n}$ for nonnegative $n$ are defined by ordinary differential equations of the first order; all other variables $v_{n}$ and $r_{n}$ are only arithmetic combinations in terms of the sets $\left\{q_{n}\right\}$ and $\left\{f_{n}\right\}$. From Eqs. (3.12), one sees that the zonal damping rate of the forced signal is proportional to $\sqrt{\alpha \gamma}$, rather than being characterized by either $\alpha$ or $\gamma$. When either the Rayleigh friction rate or the Newtonian cooling rate is very small, the forced signals are expected to reach far away from the heating region in the zonal direction.

Equations (3.12a) and (3.12c) are solved in a zonally cyclic domain. It is common to use Fourier series to represent all the variables and the forcing and then determine the coefficients. Here, we use the Green's function method to obtain the solutions to Eqs. (3.12a) and (3.12c). The Green's function method is outlined in appendix B. The solutions to Eqs. (3.12a) and (3.12c) are therefore expressed as

$$
\begin{gathered}
q_{0}(x)=\int_{0}^{C} G_{0}\left(x, x_{0}\right)\left[-\sqrt{\alpha} f_{0}\left(x_{0}\right)\right] d x_{0} \quad \text { and } \quad(3.13 \mathrm{a}) \\
q_{n+1}(x)=\int_{0}^{C} G_{n+1}\left(x, x_{0}\right) \frac{\sqrt{\alpha}}{2}\left[f_{n-1}\left(x_{0}\right)+2 n f_{n+1}\left(x_{0}\right)\right] d x_{0} \\
\quad \text { for } n \geq 1,
\end{gathered}
$$

where $G_{0}\left(x, x_{0}\right)$ and $G_{n+1}\left(x, x_{0}\right)$ are given in appendix $\mathrm{B}$, and $C$ is the length of a cyclic domain. When $C \rightarrow$ 
TABLE 1. Scaling units for different vertical modes. The first column is the gravity wave speeds of the modes; the second column is the length scales; the third column is the timescales; the fourth column is the nondimensional earth's circumference in terms of the length scales; and the fifth column is the nondimensional damping rate corresponding to $1 /(4.0$ days $)$.

\begin{tabular}{ccccc}
\hline \hline $\begin{array}{c}\sqrt{g h_{m}} \\
\left(\mathrm{~m} \mathrm{~s}^{-1}\right)\end{array}$ & $\begin{array}{c}{\left[L_{m}\right]} \\
(\text { degrees })\end{array}$ & $\begin{array}{c}{\left[T_{m}\right]} \\
(\text { days })\end{array}$ & {$\left[C_{m}\right]$} & $\begin{array}{c}\text { Damping } \\
\text { rate }\end{array}$ \\
\hline 20.0 & 8.4 & 0.53 & 42.9 & 0.133 \\
28.2 & 10.0 & 0.46 & 36.0 & 0.114 \\
60.0 & 14.6 & 0.31 & 24.7 & 0.078 \\
\hline
\end{tabular}

$\infty$, solutions $(3.13 \mathrm{a}, \mathrm{b})$ become the solutions in a zonally infinite domain.

\section{The response to an isolated heat source}

In this section solutions to the shallow-water system in a zonally cyclic domain and in a zonally infinite domain will be found for the various combinations of Rayleigh friction and Newtonian cooling. We will illustrate how the solutions are affected by the dissipation, the zonal domain, and the vertical heating structure (represented by the gravity wave speed). Since the scaling factors $[L]$ and $[T]$ are different for different vertical modes, we select three different sets of units that correspond to the vertical modes with the gravity wave speed of 20,28, and $60 \mathrm{~m} \mathrm{~s}^{-1}$, respectively. Some of the scaling values are listed in Table 1. Although these values are arbitrarily selected, the gravity wave speeds of 20 and $60 \mathrm{~m} \mathrm{~s}^{-1}$ are prominently represented by a true deep heating profile in Fig. $2 b$, which is representative for the heating by deep convection in the Tropics, ${ }^{1}$ typical for communicating the response of the system a heat source whose vertical structure is given by Fig. $2 b$.

To compare directly with simple solutions in Gill (1980), the external forcing is prescribed slightly differently from what is expressed by Eq. (A.1) in the zonal direction. The horizontal structure of the heating in this section has the form

$$
F(x, y)=f(x) \exp \left(-\frac{y^{2}}{2 Y^{2}}\right),
$$

where $Y$ is $1100 \mathrm{~km}$ (corresponding to $10^{\circ}$ of latitude) and

$$
f(x)= \begin{cases}\cos \left(\frac{x-x_{c}}{x_{r}-x_{l}} \pi\right) & x_{l} \leq x \leq x_{r} \\ 0 & 0<x<x_{l} \text { or } \\ & C \geq x>x_{r} ;\end{cases}
$$

\footnotetext{
${ }^{1}$ The full solution is the integral over the whole spectrum. The full numerical solutions and their three-dimensional structures corresponding to many cases discussed in this paper can be found in Wu et al. (2000a).
}

TABLE 2. The list of parameters for each case. The second column is the gravity wave speeds of the modes; the third column is the damping timescales for Rayleigh friction; the fourth column is the damping timescales for Newtonian cooling; the fifth column is the forcing types for each case; and the sixth column is the domain types for each case.

\begin{tabular}{lcrrll}
\hline \hline & $\begin{array}{c}\sqrt{g h_{m}} \\
\left(\mathrm{~m} \mathrm{~s}^{-1}\right)\end{array}$ & $\begin{array}{c}1 / \alpha \\
(\text { days })\end{array}$ & $\begin{array}{c}1 / \gamma \\
(\text { days })\end{array}$ & $\begin{array}{c}\text { Forcing } \\
\text { type }\end{array}$ & $\begin{array}{c}\text { Zonal } \\
\text { domain }\end{array}$ \\
\hline Case I & 28.2 & 4.0 & 4.0 & Symmetric & Cyclic \\
Case II & 20.0 & 4.0 & 4.0 & Symmetric & Cyclic \\
Case III & 60.0 & 4.0 & 4.0 & Symmetric & Cyclic \\
Case IV & 60.0 & 10.0 & 10.0 & Symmetric & Cyclic \\
Case V & 60.0 & 10.0 & 10.0 & Symmetric & Unbounded \\
Case VI & 60.0 & 1000.0 & 10.0 & Symmetric & Cyclic \\
Case VII & 60.0 & 10.0 & 100.0 & Symmetric & Cyclic \\
Case VIII & 60.0 & 1000.0 & 10.0 & Symmetric & Unbounded \\
Case IX & 60.0 & 10.0 & 100.0 & Symmetric & Unbounded \\
Case X & 60.0 & 1000.0 & 10.0 & Asymmetric & Cyclic \\
Case XI & 60.0 & 10.0 & 100.0 & Asymmetric & Cyclic \\
\hline
\end{tabular}

here $x_{l}, x_{c}, x_{r}$ correspond to the longitudes $80^{\circ}, 100^{\circ}$, $120^{\circ} \mathrm{E}$, respectively; $C$ is the circumference of the earth.

Four categories of damping cases are examined: 1) the strong-damping cases in which the damping timescales for Rayleigh friction and Newtonian cooling are prescribed as 4 days, 2) the weak-damping cases in which the damping timescales for Rayleigh friction and Newtonian cooling are prescribed as 10 days, 3) the Newtonian cooling-dominant cases in which the damping timescale is 1000 days for Rayleigh friction and 10 days for Newtonian cooling, and 4) the Rayleigh friction-dominant cases in which the damping timescale is 10 days for Rayleigh friction and 100 days for Newtonian cooling. Since the solutions in a zonally cyclic domain may differ significantly from those in a zonally infinite domain, we will carry out the calculations in both types of zonal domains for each case. Those cases that will be analyzed in the following are listed in Table 2.

\section{a. Responses under strong damping}

In case I, the gravity wave speed of $28.2 \mathrm{~m} \mathrm{~s}^{-1}$ and the damping timescales of 4 days for Rayleigh friction and Newtonian cooling are prescribed. These prescribed parameters are almost identical to those given in Gill (1980) provided that $10^{\circ}$ is taken as the equivalent to one spatial unit in Gill (1980). In this case, the external forcing projects only onto the zero-order parabolic cylinder function, and horizontal structure of response (not shown here) is almost identical to Fig. 1 in Gill (1980), which is anticipated since the fastest propagating Kelvin signal with a propagation speed of $28.2 \mathrm{~m} \mathrm{~s}^{-1}$ takes about 16 days to travel along the earth's circumference, and with a 4-day damping timescale, the forced signal is diminishingly small after it travels three quarters of the earth's circumference. Therefore, a zonally cyclic domain in this case does not have much impact on the solution.

Figure 3 shows the response corresponding to a ver- 

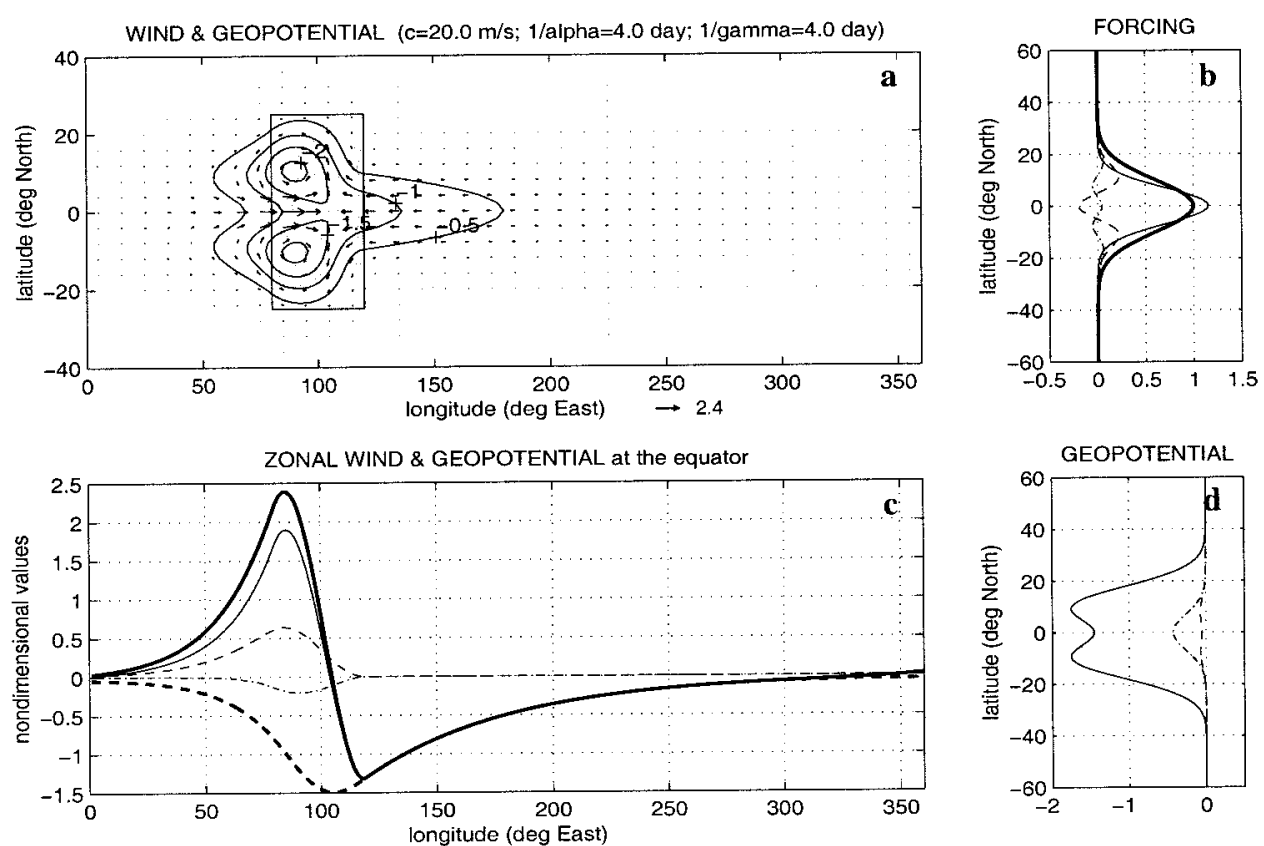

FIG. 3. The solutions for case II (strong Rayleigh friction and Newtonian cooling, cyclic domain, $c=20$ $\mathrm{m} \mathrm{s}^{-1}$ ). (a) The vector field (with its scale shown under the panel) of horizontal velocities are superposed on the contoured geopotential. The shaded area is where the heat is applied. (b) Plot of the meridional profile of the heating (the bold solid line). (c) Plot of the total zonal velocity at the equator (the bold solid line), the total geopotential at the equator (the bold dashed line), and the decomposed form of the zonal velocity at the equator [The contributions from meridional modes $\psi_{0}(y), \psi_{2}(y)$, and $\psi_{4}(y)$ at the equator are represented by the thin solid line, the thin dashed line, and the thin dash-dotted line, respectively]. (d) Plot of the meridional structures of the geopotential at different longitudes (solid line: $100^{\circ} \mathrm{E}$ corresponding to the heating center; dashed line: $10^{\circ} \mathrm{E}$; and dash-dotted line: $190^{\circ} \mathrm{E}$ ).

tical mode whose horizontal gravity wave speed is 20 $\mathrm{m} \mathrm{s}^{-1}$ (case II, strong dampings, cyclic domain). In this case, the forcing projects onto higher-order parabolic cylinder functions as well (Fig. 3b). Thus, the response accounted for by the higher modes is no longer zero everywhere (see the dash-dotted line in Fig. 3c). The forced signals propagate at smaller speeds; their corresponding spatial decay rates are larger than those in case I; and therefore the horizontal structure of the response (Fig. 4a) looks like a zonally squeezed version of the Gill solution. Since the length scale $[L]$ in this case is smaller than that in the previous case, the Kelvin signal to the east of the heating has a smaller meridional scale and is more equatorially trapped.

For a vertical mode of larger vertical wavelength and faster horizontal propagating speed, the length scale $[L]$ is larger. The corresponding parabolic cylinder functions are also of larger meridional scales. Again, many parabolic cylinder functions are needed to expand the forcing given by Eq. (4.1). The solutions for the case where the horizontal gravity wave speed is $60 \mathrm{~m} \mathrm{~s}^{-1}$ (case III) are shown in Fig. 4. Clearly, the forced signals cover a larger meridional extent. Since it takes less than 8 days for a Kelvin signal in this case to travel along the earth's circumference, the eastward propagating Kelvin signal and westward propagating Rossby signals overlap in the most of the equatorial zone.

\section{b. Responses under weak damping}

When dissipation is weak, the forced signals can propagate farther away from the heating region before they are damped. In a cyclic domain, this means that a forced signal may still keep large enough amplitude after it travels around the earth's circumference and returns to the excitation region. Figure 5 features the solutions for a vertical mode whose horizontal gravity wave speed is $60 \mathrm{~m} \mathrm{~s}^{-1}$ (case IV) in the presence of weak dissipation. Contrasting this case with the corresponding case of strong dissipation (case III, Fig. 4), one sees that the easterly winds from $30^{\circ}$ to $40^{\circ}$ latitude [mainly contributed by the modes corresponding to $\psi_{0}(y)$ and $\left.\psi_{2}(y)\right]$ are much stronger in case IV than in case III. The geopotential at the equator (Fig. 5d) and at different longitudes also shows that their zonal decay rates are very small and the geopotential is almost zonally uniform.

In a zonally infinite domain (case V, Fig. 6), the solutions are very much different from those in case IV. In the Tropics, the easterlies to the east of the heating 

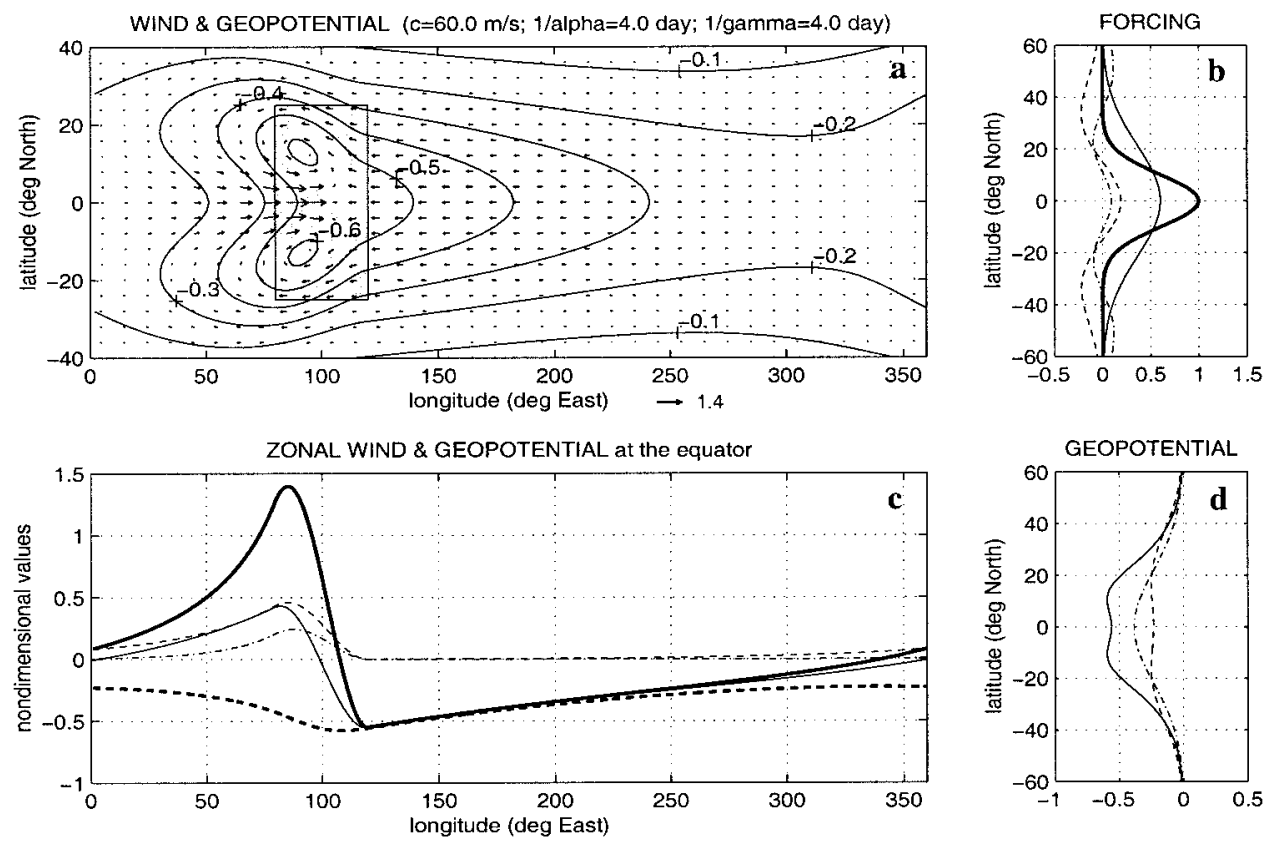

FIG. 4. The same as Fig. 3 but for case III (strong Rayleigh friction and Newtonian cooling, cyclic domain, $c=60 \mathrm{~m} \mathrm{~s}^{-1}$ ).

are weaker than those in case IV, while the westerlies near the equator are stronger than those in case IV. The geopotential is about half the amplitude of that in case IV almost everywhere. These phenomena can be explained by the lack of a strong resonant effect of the fast propagating Kelvin signal in a weakly dissipated medium that happens in case IV. In the midlatitudes, the zonal winds are much stronger and more zonally symmetric in case IV than in case V. This is exactly what one would anticipate since in case IV, the zonal winds that are associated with the Kelvin signal and $n$ $=2$ Rossby signal have the same sign and reinforce each other in addition to the resonant effect.

\section{c. The Newtonian cooling-dominant case and the Rayleigh friction-dominant case}

In the introduction, we indicated that the numerical model shows that the response is almost zonally uniform but meridionally confined in the equatorial regions when the only dissipative mechanism is Newtonian cooling. The calculation using a linear model for a Newtonian cooling-dominant case is shown in Fig. 7 (case VI). Figure 7 catches the essence of the numerical result (Fig. 1b) from a dry primitive equation model: the forced winds are almost zonally uniform and meridionally confined to the heating latitudes. In this case, the meridional scale (the effective equatorial radius of deformation) of the parabolic cylinder functions $\psi_{n}(y)$ expressed by Eq. (3.3) is very small. Hence, the Kelvin signal and loworder Rossby signals do not extend to high latitudes. From Fig. 7b, one sees that the heating mainly projects onto the modes $\psi_{n}(y)$ with their turning points residing inside the meridional extent of the heating. Thus, the response to the heating is also accounted for by these modes and confined to the heating latitudes. Since the zonal decay rates (proportional to $\sqrt{\alpha \gamma}$ ) are all so small for the Rossby signals, the zonal wind response accounted for by those Rossby modes are almost zonally uniform outside the heating longitudes (Fig. 7c), and the geopotential is zonally uniform at every latitude (Fig. 7d).

The maximum zonal winds at about $15^{\circ}$ latitude are mainly contributed by the easterlies associated with $\psi_{2}(y)$. In a very narrow zone along the equator, the forced zonal response associated with that mode is westerly winds, which counteract the easterlies associated with $\psi_{0}(y)$ and result in the easterlies to the east of the heating and westerlies to the west of the heating. The effective equatorial radius of deformation of the shallow-water system becomes smaller when Rayleigh friction is smaller; therefore, the zonal wind response is a meridionally shrunk form (not shown here) of Fig. 7a with modes of larger $n$ accounting for a slightly larger amount of the response.

Figure 8 shows the results when Rayleigh friction is the dominant dissipative mechanism for a vertical mode whose horizontal gravity wave speed is $60 \mathrm{~m} \mathrm{~s}^{-1}$ (case VII). Clearly, the wind field (Fig. 8a) retains most of the characteristics of Fig. 1a. In this case, the meridional scale (the effective equatorial radius of deformation) of the parabolic cylinder functions $\psi_{n}(y)$ expressed by Eq. (3.3) is very large. Hence, all the Kelvin and Rossby signals extend to high latitudes. Near the equator, the 

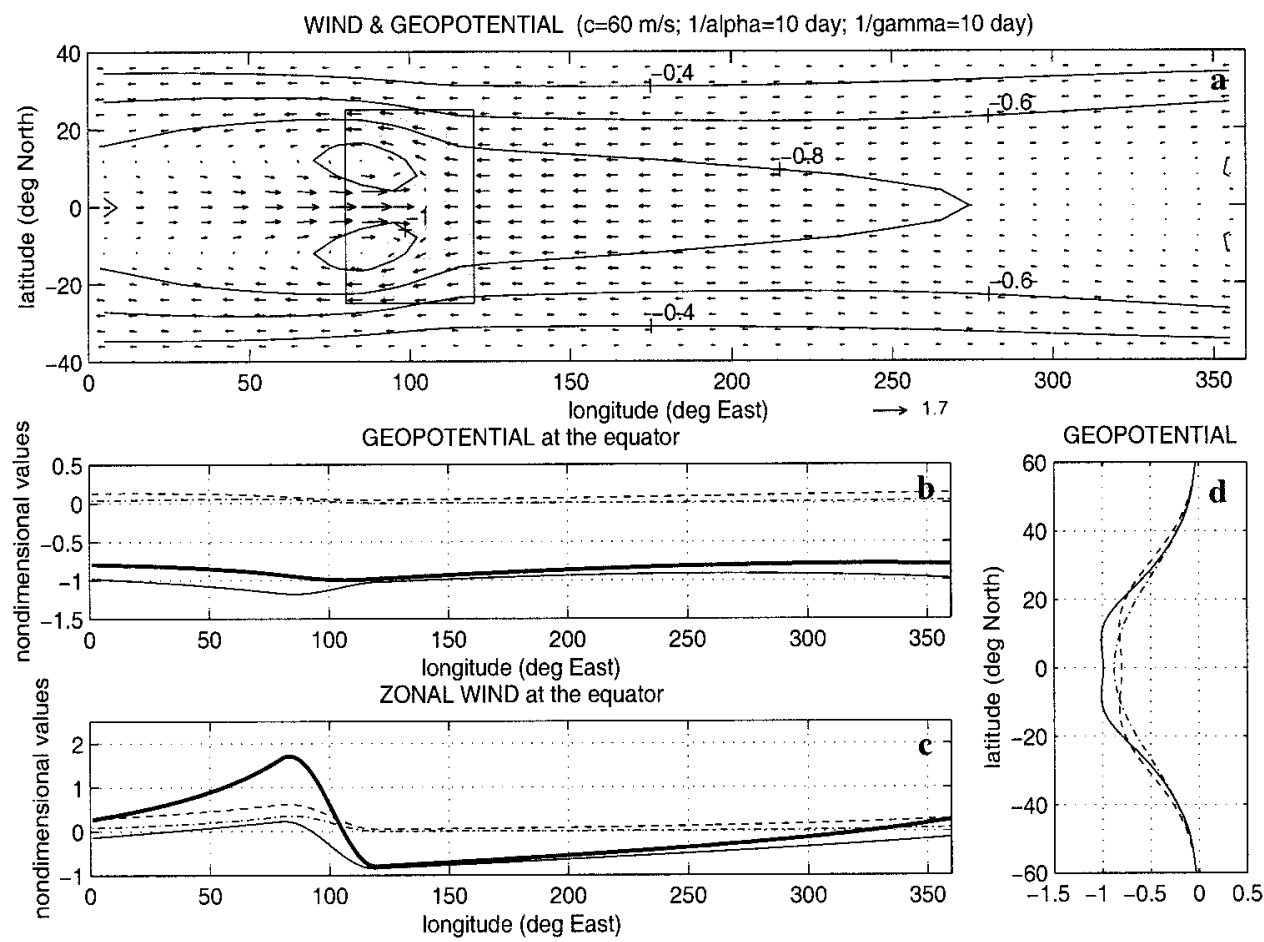

FIG. 5. The solutions for case IV (weak Rayleigh friction and Newtonian cooling, cyclic domain, $c=60$ $\mathrm{m} \mathrm{s}^{-1}$ ). (a) The vector field (with its scale shown under the panel) of horizontal velocities are superposed on the contoured geopotential. The shaded area is where the heating happens. (b) Plot of the total geopotential at the equator (the bold solid line), and the decomposed form of the geopotential at the equator (the contributions from meridional modes $\psi_{0}(y), \psi_{2}(y)$, and $\psi_{4}(y)$ at the equator are represented by the thin solid line, the thin dashed line, and the thin dash-dotted line, respectively). (c) Plot of the total zonal velocity at the equator (the bold solid line), and the decomposed form of the zonal velocity at the equator [The contributions from meridional modes $\psi_{0}(y), \psi_{2}(y)$, and $\psi_{4}(y)$ at the equator are represented by the thin solid line, the thin dashed line, and the thin dash-dotted line, respectively]. (d) Plot of the meridional structures of the geopotential at different longitudes (solid line: $100^{\circ} \mathrm{E}$ corresponding to the heating center; dashed line: $10^{\circ} \mathrm{E}$; and dash-dotted line: $\left.190^{\circ} \mathrm{E}\right)$.

zonal wind signal associated with the Kelvin mode [with meridional structure $\left.\psi_{0}(y)\right]$ and the zonal wind signal associated with the Rossby modes are of the opposite signs and cancel each other, and the net winds are easterlies to the east of the heating and westerlies to the west of the heating. However, in the midlatitudes, the zonal wind signals associated with the Kelvin mode and the second Rossby mode have the same signs (see the thin solid, dashed, and dash-dotted lines in Figs. 8b,c); therefore, the almost zonally uniform easterlies are seen. At higher latitudes, the contribution from the Kelvin signal is diminishingly small, but the zonal wind signals associated with different Rossby modes [e.g., $\psi_{2}(y)$ and $\left.\psi_{4}(y)\right]$ have opposite signs and cancel each other. The meridional wind signals are an order of magnitude smaller than the zonal wind signals almost everywhere. The geopotential, of course, is zonally uniform and meridionally extended (Fig. 8d).

When a smaller Newtonian cooling rate is selected, the results and arguments are essentially similar to the above case. However, the meridional scale of $\psi_{n}(y)$ becomes larger. The wind field (not shown here) outside the equatorial zone looks like a meridionally stretched form of Fig. 8a.

The solutions for both the Newtonian cooling-dominant case (case XIII) and the Rayleigh friction-dominant case (case IX) in an infinite zonal domain are shown in Fig. 9. It is clear that the responses in both cases are significantly different from those in a zonally cyclic domain. In both cases, the Kelvin signal extends to the east of the heating and the Rossby signals extend to the west of the heating. However, since the effective equatorial radius of deformation is proportional to the fourth root of the Prandtl number (the ratio between Rayleigh friction rate and Newtonian cooling rate), the meridional extent of the response in the Rayleigh friction-dominant case is much larger than that in the Newtonian coolingdominant case.

The above cases are for heating symmetric about the equator. To test whether the characteristics described above also hold for the circulations driven by aymmetric heating, two more calculations are carried out. In these two cases, the heat source applied in previous cases is moved $10^{\circ}$ northward. Figure 10 shows the forced cir- 

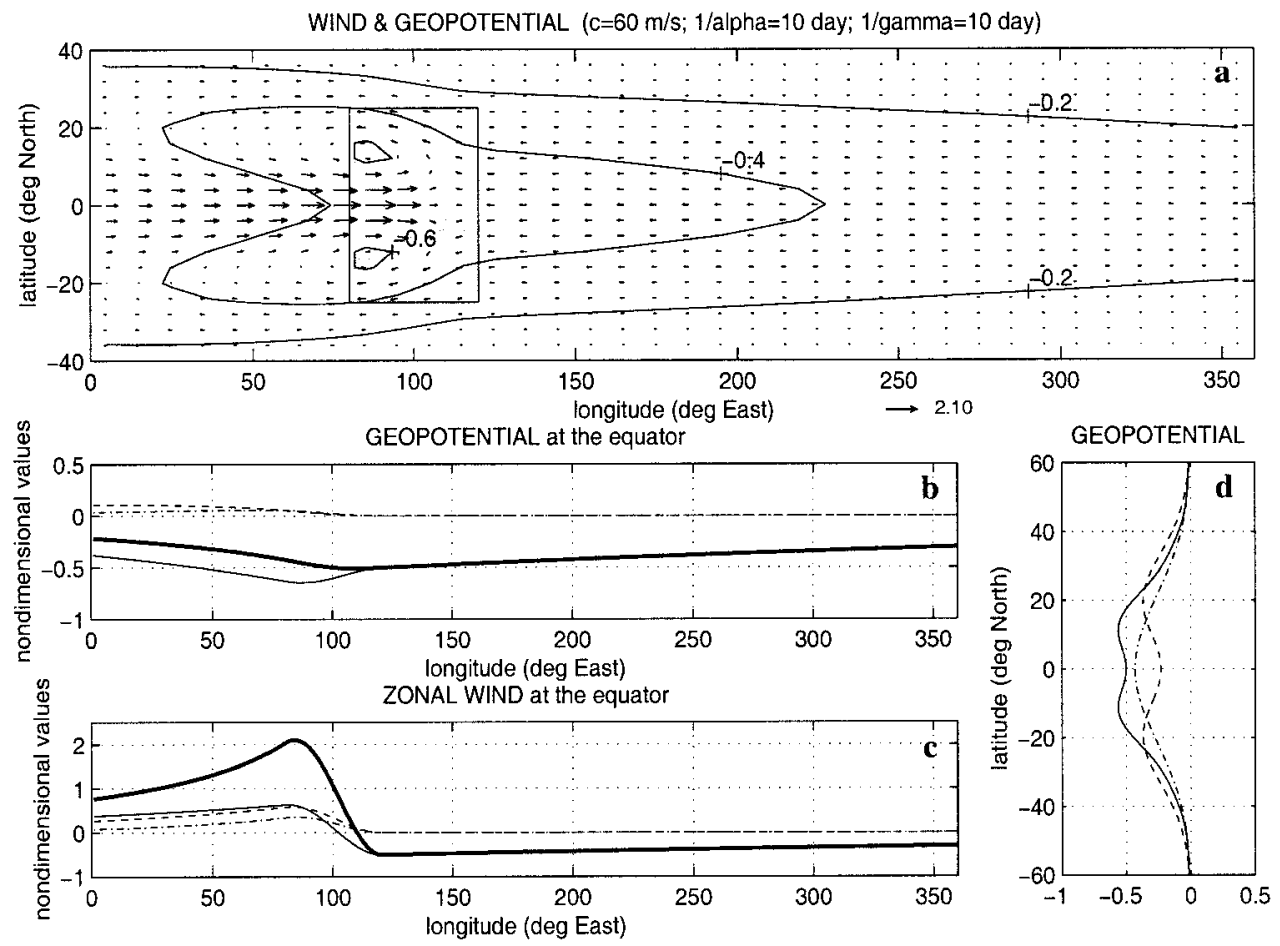

FIG. 6. The same as Fig. 5 but for case V (weak Rayleigh friction and Newtonian cooling, unbounded domain, $c=60 \mathrm{~m} \mathrm{~s}^{-1}$ ).

culations for both the Newtonian cooling-dominant case (case X, Figs. 10a,b) and the Rayleigh friction-dominant case (case XI, Figs. 10c,d). Comparing all the panels of Fig. 10 with the corresponding panels of Fig. 7 and Fig. 8, one would easily conclude that circulations driven by asymmetric heating have many similar characteristics to those driven by symmetric heating in both cases: the forced circulations are still quite zonally symmetric, but meridionally confined when Newtonian cooling dominates and meridionally extended when Rayleigh friction dominates.

\section{Implications}

The theoretical solutions obtained in the previous sections may explain some observed phenomena. In this section, we will explore the implications of these theoretical results to the thermally driven surface winds and for the zonally uniform low-frequency variation in pressure and temperature in the Tropics.

\section{a. Thermally driven surface winds in the Tropics}

From Eqs. (2.3a-d), one can derive a single equation of mass-weighted meridional velocity $\nu$ for the steady case:

$$
\begin{aligned}
& \frac{\gamma}{N^{2} \alpha}\left(\frac{\partial^{2}}{\partial z^{2}}-\frac{1}{4 H^{2}}\right)\left(\beta^{2} y^{2}+\alpha^{2}\right) v \\
& \quad+\left[\left(\frac{\partial^{2}}{\partial x^{2}}+\frac{\partial^{2}}{\partial y^{2}}\right)+\frac{\beta}{\alpha} \frac{\partial}{\partial x}\right] v=\mathcal{H},
\end{aligned}
$$

where

$$
\mathcal{H}=\left(\beta y \frac{\partial}{\partial x}-\alpha \frac{\partial}{\partial y}\right) \frac{1}{N^{2} \alpha}\left(\frac{\partial}{\partial z}-\frac{1}{2 H}\right) Q .
$$

Equation (5.1) is an elliptic equation. When either $\alpha$ $\rightarrow 0$ or $\gamma \rightarrow 0$, or both $\alpha$ and $\gamma$ are small, as it was shown in the previous section,

$$
\partial / \partial x \rightarrow 0 .
$$

Using Eq. (5.2), one can further obtain an approximation for Eq. (5.1):

$$
\frac{\gamma}{N^{2} \alpha}\left(\frac{\partial^{2}}{\partial z^{2}}-\frac{1}{4 H^{2}}\right)\left(\beta^{2} y^{2}+\alpha^{2}\right) v+\frac{\partial^{2} v}{\partial y^{2}}=\mathcal{H} .
$$

For an elliptic equation of two spatial dimensions like Eq. (5.3), one would have an integral property as

$$
\int_{y} \int_{z} v(z, y) d z d y=I,
$$

where $I$ is an integral form of external forcing term $\mathcal{H}$. 

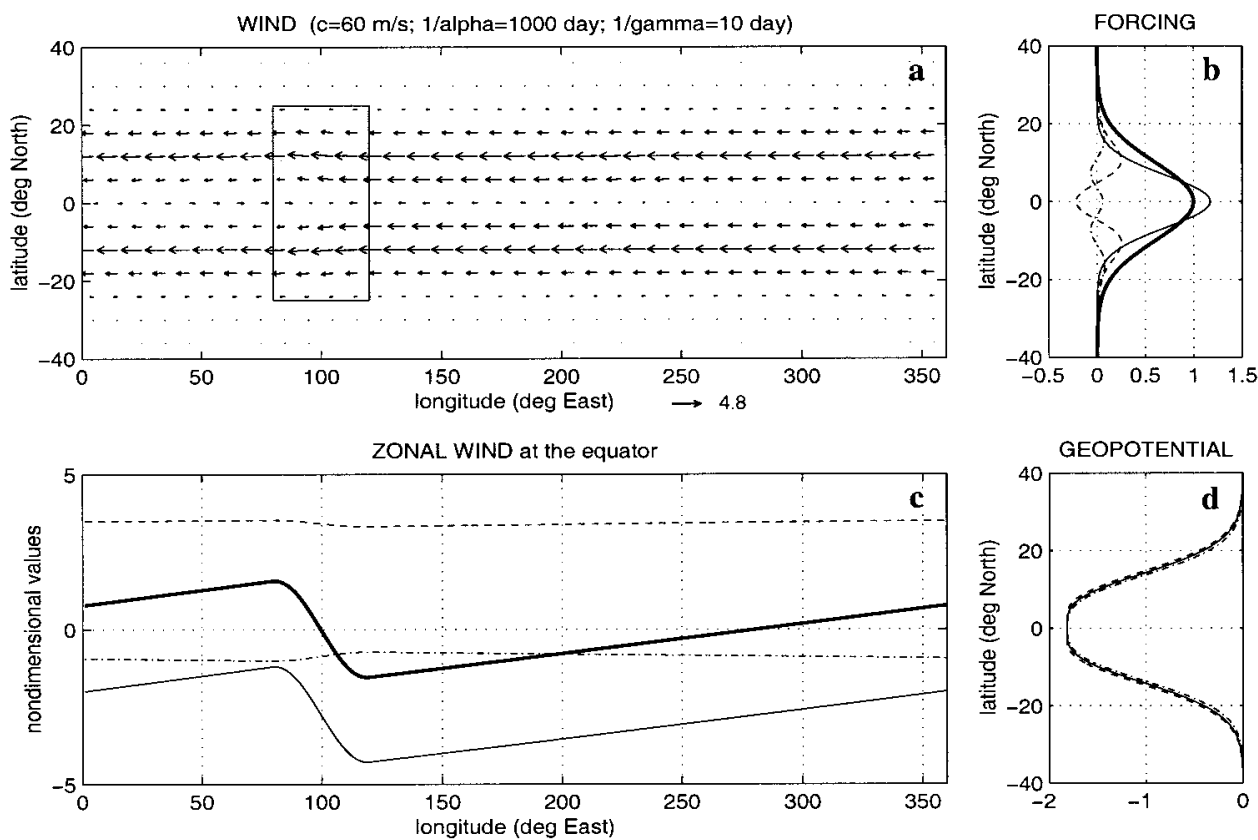

FIG. 7. The solutions for case VI (the Newtonian cooling-dominant case, cyclic domain, $c=60 \mathrm{~m} \mathrm{~s}^{-1}$ ). (a) Plot of the vector field (with its scale shown under the panel) of horizontal velocities. The shaded area is where the heating happens. (b) Plot of the meridional profile of the heating (the bold solid line) and its decomposed form in terms of the parabolic cylinder functions with characteristic meridional scale of the shallow-water system. The parts associated with $\psi_{0}(y), \psi_{2}(y)$, and $\psi_{4}(y)$ are shown by the thin solid line, the thin dashed line, and the thin dash-dotted line, respectively. (c) Plot of the total zonal velocity at the equator (the bold solid line), and the decomposed form of the zonal velocity at the equator [The contributions from meridional modes $\psi_{0}(y), \psi_{2}(y)$, and $\psi_{4}(y)$ at the equator are represented by the thin solid line, the thin dashed line, and the thin dash-dotted line, respectively]. (d) Plot of the meridional structures of the geopotential at different longitudes (solid line: $100^{\circ} \mathrm{E}$ corresponding to the heating center; dashed line: $10^{\circ} \mathrm{E}$; and dash-dotted line: $\left.190^{\circ} \mathrm{E}\right)$.
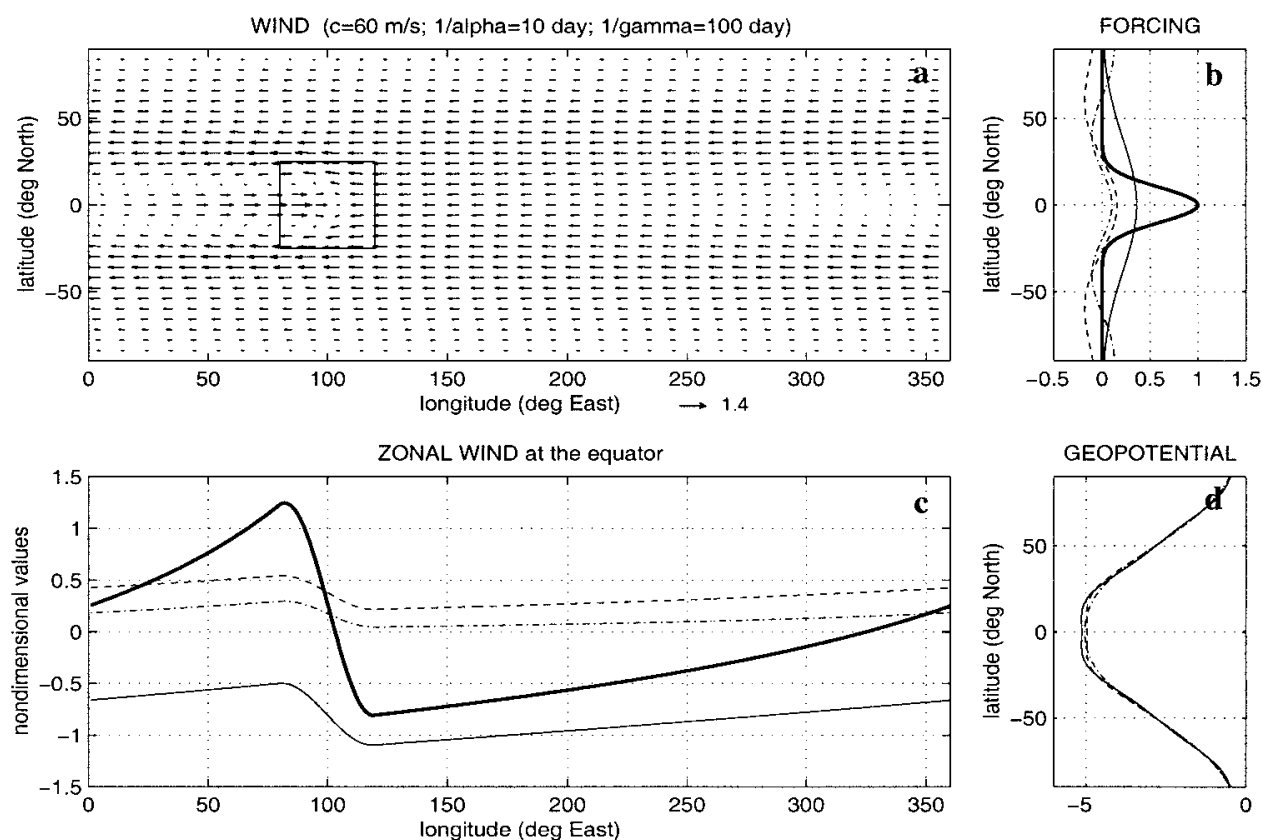

FIG. 8. The same as Fig. 7 but for case VII (the Rayleigh friction-dominant case, cyclic domain,

$$
c=60 \mathrm{~m} \mathrm{~s}^{-1} \text { ). }
$$



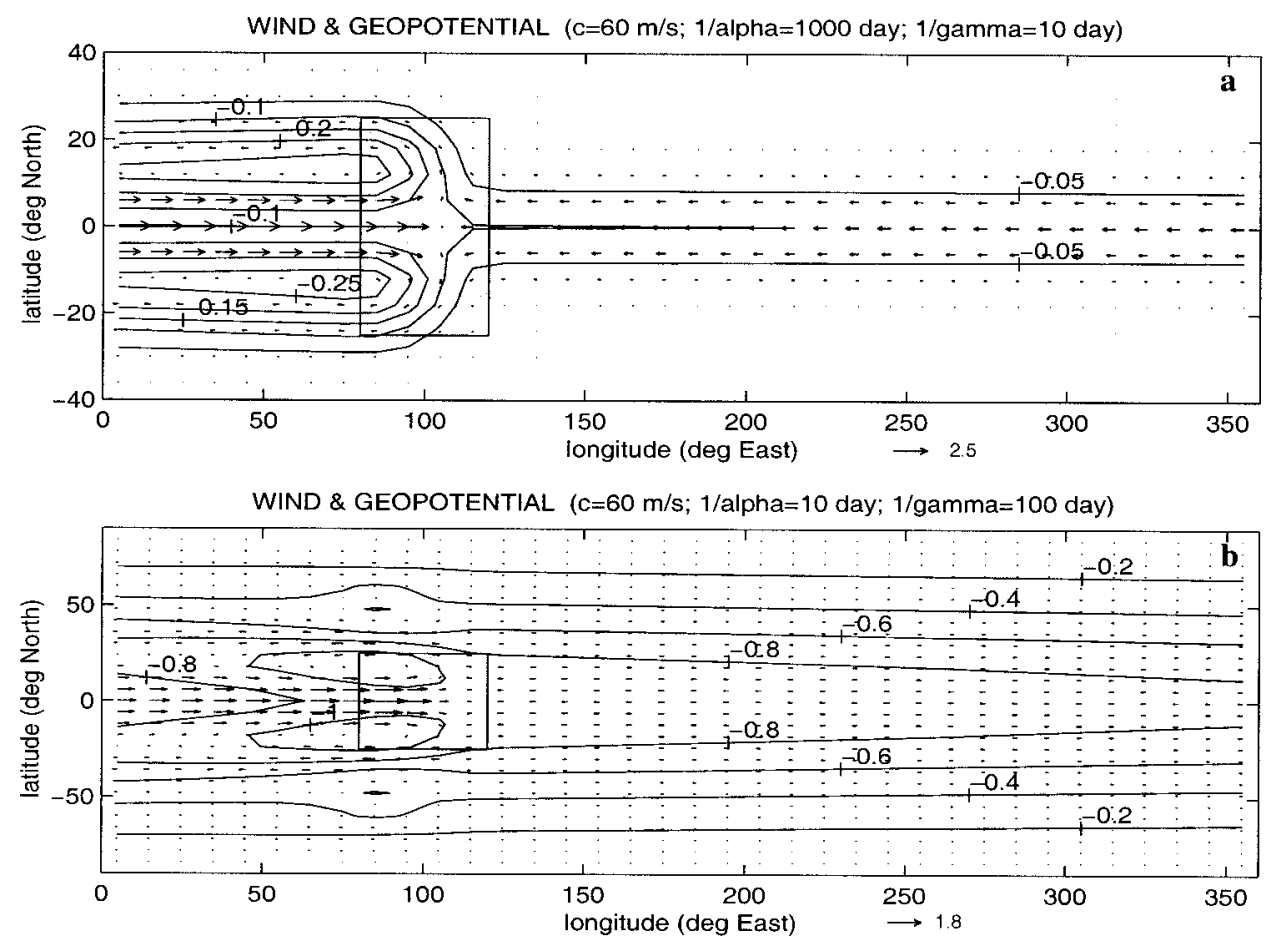

FIG. 9. The solutions for (a) case VIII (the Newtonian cooling dominant case, unbounded domain, $c=$ $60 \mathrm{~m} \mathrm{~s}^{-1}$ ), and (b) case IX (the Rayleigh friction dominant case, unbounded domain, $c=60 \mathrm{~m} \mathrm{~s}^{-1}$ ). In both panels, the vector field (with its scale shown under the panel) of horizontal velocities are superposed on the contoured geopotential. The nondimensional contour interval is 0.05 for case VIII and 0.2 for case IX.
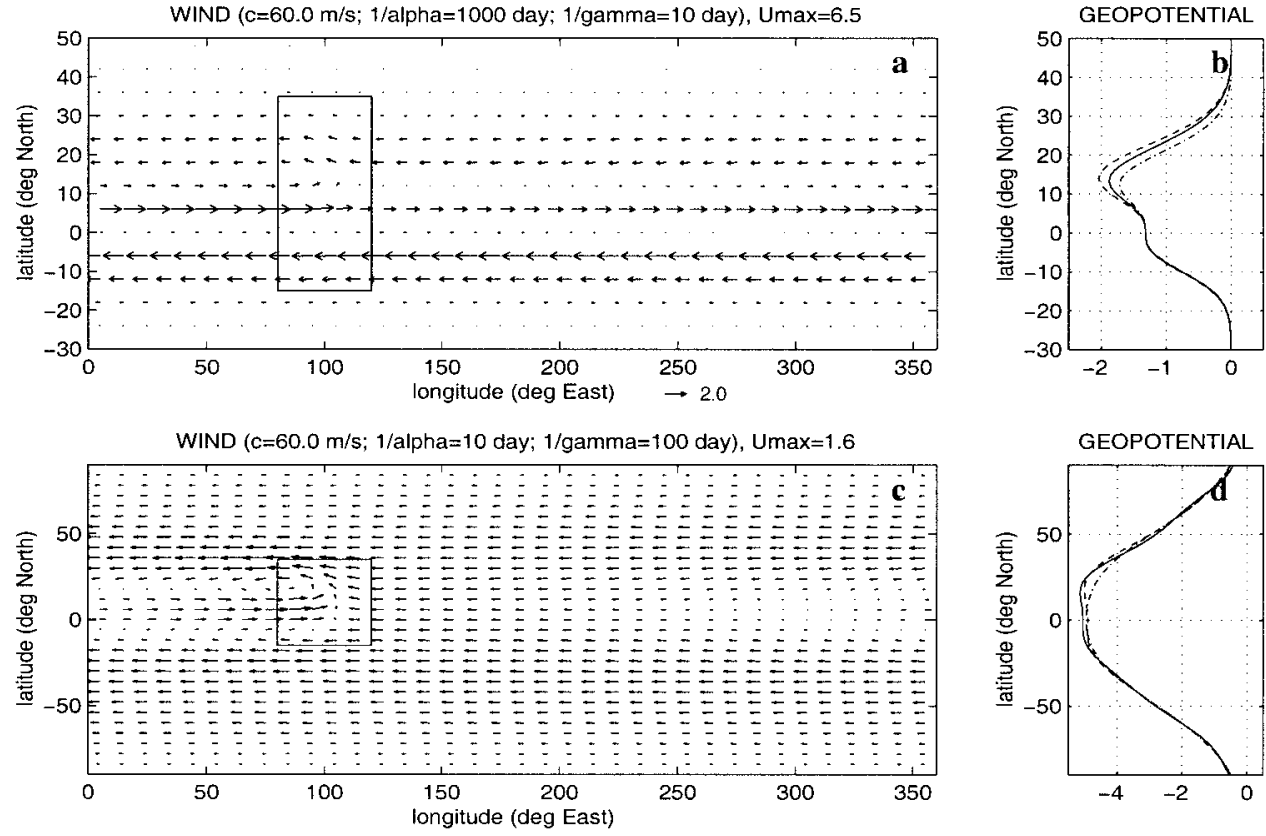

FIG. 10. The solutions for case $\mathrm{X}$ [the Newtonian cooling dominant case, cyclic domain, $c=60 \mathrm{~m} \mathrm{~s}^{-1}$, asymmetric heating, (a) and (b)] and case XI [the Rayleigh friction dominant case, cyclic domain, $c=60$ $\mathrm{m} \mathrm{s}^{-1}$, asymmetric heating, (c) and (d)]. In (a) and (c), the vector field (with their scales shown under each panel) of horizontal velocities are plotted. The shaded area is where the heating happens. (b) and (d) Meridional structures of the geopotential at different longitudes (solid line: $100^{\circ} \mathrm{E}$ corresponding to the heating center; dashed line: $10^{\circ} \mathrm{E}$; and dash-dotted line: $190^{\circ} \mathrm{E}$ ). 
When the external forcing is localized, the right-hand side of Eq. (5.4) is limited. Physically, Eqs. (5.3) and (5.4) indicate that when the solution $\nu$ is meridionally confined, it must be vertically extended, and vice versa.

Since the forced response to an elevated local thermal source is meridionally extended when Rayleigh friction dominates and is meridionally confined when Newtonian cooling dominates, one expects significant forced circulations below the bottom of the heating in the Newtonian cooling-dominant case and little response below the heating in the Rayleigh friction-dominant case as Fig. 1 shows. More detailed numerical results for this argument can be found in $\mathrm{Wu}$ et al. (2000a).

For the case when both $\alpha$ and $\gamma$ are large, a similar argument still holds since an integral property similar to Eq. (5.4) is intrinsic to an elliptic equation of any spatial dimensions. The strong momentum damping and thermal damping only make the forced response more zonally confined and, hence, more vertically extended. Therefore, as long as thermal damping dominates, an elevated heat source would drive strong surface circulations. A recent study by Chiang et al. (2001) provides supportive evidence for this argument. In Chiang et al. (2001), it is shown that the surface response to an elevated heating is very sensitive to the thermal relaxation rate of the planetary boundary layer (PBL). When the thermal relaxation rate of PBL is large, the surface response is strong, and when the thermal relaxation rate of PBL is small, the surface response is weak.

\section{b. Zonally symmetric low-frequency perturbations in the Tropics}

An interesting feature of the tropical atmosphere that has not been well explained is the zonally uniform lowfrequency variation in pressure and temperature in the Tropics. Barnett (1985) analyzed the variations in the 200-hPa height field and showed that the variations appear coherent for the tropical belt around the entire globe. Newell and Wu (1992) found that the correlation between the tropospheric temperature in the whole tropical zone and the averaged tropospheric temperature in the tropical eastern Pacific is almost zonally uniform. Yulaeva and Wallace (1994) and Wallace et al. (1998) further observed that the warming signals are highly uniform in the zonal direction and meridionally extended. These results indicate that the locally forced warming signal exhibits little spatial decay when it propagates away from the heating region, which can hardly be explained by the structure of the classical Gill solutions.

In the upper half of the tropical troposhpere, although there is no consensus of the value of the Rayleigh friction rate and the Newtonian cooling rate, the thermal damping timescale is believed to be of a few tens of days and the momentum damping is even smaller and often neglected (Jin and Hoskins 1995; Rodwell and Hoskins 1996). Hence, the zonal damping timescale is very long. We have also shown, in the previous sections, that deep heating projects significantly onto the fast propagating vertical modes. For these fast propagating modes, under such weak dissipation, the geopotential response hardly decays in the zonal direction in a zonally cyclic domain. Hence, these observed features can somehow be explained by the linear response of the tropical atmosphere to deep heating due to cumulus convection. However, it remains to be further examined whether the observed zonal mean signal is solely due to the linear response to an isolated heat source in a zonally cyclic domain.

\section{Discussion and conclusions}

In the previous sections, the forced shallow-water system resulting from the vertical decomposition of a vertically semi-infinite atmosphere was analytically solved in the presence of different combinations of Rayleigh friction and Newtonian cooling. Both zonally cyclic and zonally infinite domains were considered to examine the sensitivity of the horizontal structure of the forced response to the various model domains. The solutions were then used to understand both the thermally driven surface winds and the zonally uniform low-frequency variation in pressure and temperature in the Tropics.

The meridional extent of the forced response is characterized by the effective equatorial radius of deformation of a shallow-water system with a given equivalent depth, which is the equatorial radius of deformation of the shallow-water system multiplied by the fourth root of the Prandtl number (the ratio between the Rayleigh friction rate and the Newtonian cooling rate). When the values of the Rayleigh friction rate and the Newtonian cooling rate are same, the effective equatorial radius of deformation of a shallow-water system is exactly the equatorial radius of deformation of that shallow-water system. Hence, the forced response covers a larger meridional domain for a shallow-water system with larger equivalent depth compared to those with relatively smaller equivalent depth under external forcing of the same meridional structure. In Wu et al. (2000b), we showed that the deeper the heating, the larger the portion of heating energy accounted for by the modes with large equivalent depth. Hence, the deep heating implies that the forced atmospheric circulation covers a large meridional extent.

When the values of the Rayleigh friction rate and the Newtonian cooling rate are different, the effective equatorial radius of deformation of a shallow-water system will be very large when Rayleigh friction dominates and very small when Newtonian cooling dominates. The turning point of any single parabolic cylinder function can be far outside the heating latitude in the Rayleigh friction-dominant case, and therefore, the total forced response can extend to a very high latitude. For the Newtonian cooling-dominant case, 
however, almost all the parabolic cylinder functions onto which the forcing has significant projection have their turning points inside the latitudinal boundaries of the heating, and the total response is confined to the heating latitudes. The more extensive numerical results from a dry primitive equation model have already been shown in $\mathrm{Wu}$ et al. (2000a), where the implications of these results to the thermally driven surface winds in the Tropics are emphasized.

The zonal decay scale of a shallow-water system is characterized by the product of the gravity wave speed of the shallow-water system and the damping timescale. When this product is small enough, the solutions in a zonally infinite domain can be very good approximations to the solutions in a zonally cyclic domain. Since deep thermal forcing generally projects a significant part of its energy onto the shallow-water system with a relatively large gravity wave speed, a significant amount of the forced signals can propagate a great distance along the equator before being totally damped and therefore can interfere with the signal coming from the opposite direction. In such a case, the Kelvin signal and Rossby signals can either interfere with or reinforce each other. The net results in a zonally cyclic domain are therefore, in general, different from those in an unbounded domain in which only the Kelvin signal is seen to the east of the heating and only Rossby signals are seen to the west of the heating. The damping timescale is the inverse of the square root of the product of the Rayleigh friction rate and the Newtonian cooling rate. In the cases when both Rayleigh friction and Newtonian cooling are quite weak or when either Rayleigh friction or Newtonian cooling is diminishingly small, the zonal decay scale becomes very large. Hence, the response to a zonally isolated heating is almost zonally uniform outside the heating longitudes in a zonally cyclic domain.

These results provide a theoretical base for understanding the thermally driven surface winds. The linear theory states that the governing equations for the tropical atmosphere constitute an elliptical system. Therefore, when the response to a local elevated heat source is horizontally confined, it must be vertically extended. This means that significant surface winds are driven by an elevated heat source in the case when thermal damping dominates. However, when momentum damping dominates, the response is meridionally extended and is vertically confined to the heating altitudes. Therefore, surface winds cannot be driven by an elevated heat source in the case in which momentum damping dominates.

Our theoretical results also provide an explanation for the zonally uniform low-frequency variation in pressure and temperature in the upper half of the troposphere in the Tropics. Deep heating projects a significant amount of energy onto the modes with horizontal propagation speeds of several tens of meters per second. With weak momentum damping and thermal damping in the upper troposphere, the forced warming signals can travel along the earth's circumference without being significantly damped. Therefore, the zonally uniform low-frequency variation in pressure and temperature in the upper half of the troposphere in the Tropics can be, at least partly, the linear response to the local heat sources along the equatorial zone.

Acknowledgments. The lead author is grateful to Dr. D. Moore of NOAA/PMEL for several interesting and beneficial discussions. This work and publication was funded by a grant to the Hayes Center of the Joint Institute for the Study of the Atmosphere and Ocean (JI$\mathrm{SAO}$ ) at the University of Washington under NOAA Cooperative Agreement NA67RJ0155. The views expressed herein are those of the authors and do not necessarily reflect the views of NOAA or any of its subagencies.

\section{APPENDIX A}

\section{The Structure of the Prescribed Heating}

The numerical results are obtained from a dry version of the primitive equation model developed by Saravanan and McWilliams (1995). The model properties are described in Wu et al. (2000a). The prescribed heating has the following three-dimensional structure:

$$
Q=\exp \left[-\left(\lambda-\lambda_{0}\right)^{2} / \lambda_{L}^{2}\right] \exp \left(-\phi^{2} / \phi_{L}^{2}\right) F(p),
$$

where $\lambda$ is longitude, $\lambda_{0}$ is reference longitude with value of $93^{\circ} \mathrm{E}, \lambda_{L}=20^{\circ}, \phi$ is latitude, and $\phi_{L}=11^{\circ}$. For simplicity, $F(p)$ is defined as

$$
F(p)= \begin{cases}0 & p \geq p_{b} \\ F_{0} \sin \left(\frac{p-p_{t}}{p_{b}-p_{t}} \pi\right) & p_{t}<p<p_{b} \\ 0 & p \leq p_{t},\end{cases}
$$

where $p$ is pressure, $p_{b}$ is the pressure at the top of the forcing, and $p_{t}$ is the pressure at the bottom of the forcing. The cloud top is assumed at $160 \mathrm{hPa}$, and the cloud base is at $840 \mathrm{hPa}$. Here, $F_{0}$ is the maximum heating rate so that $F$ corresponds to $10 \mathrm{~mm}$ of precipitation per day.

\section{APPENDIX B}

\section{The Green's Functions in a Cyclic Domain}

In section 3, the Green's function method is used to obtain $q$, which are defined by the first-order differential equations [Eqs. $(3.12 \mathrm{a}, \mathrm{c})]$. The Green's functions defined by a first order in a cyclic domain can be constructed as the following.

Suppose that the first-order differential equation is 


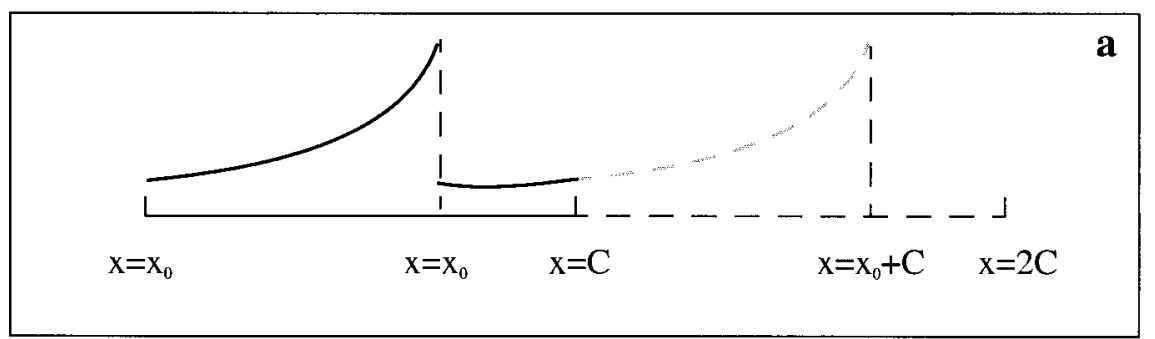

FIG. B1. The sketch of the Green's function in a cyclic domain $x \in(0, C]$ for a linear ordinary differential equation of the first order. The domain is extended to $x \in(0,2 C]$ so as to apply the jump condition at $x=x_{0}$ and the differentiable requirement at $x=C$. The Green's function is represented by the bold solid line. See also the corresponding text in appendix B.

$$
\frac{d G\left(x, x_{0}\right)}{d x}+l G\left(x, x_{0}\right)=\delta\left(x-x_{0}\right),
$$

where $x \in(0, C]$ is any position in a cyclic domain with circumference of $C$. $G$ is differentiable at everywhere except $x=x_{0}$ where an amplitude jump happens. For this reason, the Green's function $G$ in Eq. (B.1) is defined as the following:

$$
G\left(x, x_{0}\right)= \begin{cases}\tilde{G}\left(x, x_{0}\right) & \text { for } C \geq x>x_{0} \\ \tilde{G}\left(x+C, x_{0}\right) & \text { for } 0<x<x_{0},\end{cases}
$$

where $\tilde{G}\left(x, x_{0}\right)$ is defined in the domain $x \in\left(x_{0}, x_{0}\right.$ $+C]$ and is differentiable everywhere. Such a definition of the Green's function $G\left(x, x_{0}\right)$ is illustrated in Fig. B1a. Hence, the amplitude jump condition becomes

$$
\tilde{G}\left(x_{0}, x_{0}\right)-\tilde{G}\left(x_{0}+C, x_{0}\right)=1 .
$$

From Eqs. (B.1)-(B.3), one obtains

$$
G\left(x, x_{0}\right)= \begin{cases}\frac{\exp \left[-l\left(x-x_{0}\right)\right]}{1-\exp (-l C)} & \text { for } C \geq x>x_{0} \\ \frac{\exp \left[-l\left(x+C-x_{0}\right)\right]}{1-\exp (-l C)} & \text { for } 0<x<x_{0} .\end{cases}
$$

For $q_{0}$ and $q_{n+1}$ in Eqs. (3.12a) and (3.12c), the corresponding Green's functions $G_{0}\left(x, x_{0}\right)$ and $G_{n+1}\left(x, x_{0}\right)$ are of the form (B.4) with $l$ in Eqs. (B.4) being replaced by $\sqrt{\alpha \gamma}$ and $-(2 n+1) \sqrt{\alpha \gamma}$, respectively.

\section{REFERENCES}

Barnett, T. P., 1985: Three-dimensional structure of low-frequency pressure variations in the tropical atmosphere. J. Atmos. Sci. 42, 2798-2803.

Chang, C.-P., 1977: Viscous internal gravity waves and low-frequency oscillations in the tropics. J. Atmos. Sci., 34, 901-910.

Chiang, J. C. H., S. E. Zebiak, and M. A. Cane, 2001: Relative roles of elevated heating and surface temperature gradients in driving anomalous surface winds over tropical oceans. J. Atmos. Sci., in press.

Clarke, A. J., 1994: Why are surface equatorial ENSO winds anomalously westerly under anomalous large-scale convection? J. Climate, 7, 1623-1627.
Dickinson, R. E., and M. A. Geller, 1968: A generalization of "Tidal theory with Newtonian cooling." J. Atmos. Sci., 25, 932-933.

Friedman, B., 1956: Principles and Techniques of Applied Mathematics. John Wiley and Sons, $315 \mathrm{pp}$.

Gill, A. E., 1980: Some simple solutions for heat induced tropical circulations. Quart. J. Roy. Meteor. Soc., 106, 447-462.

Haarsma, R. J., and J. D. Opsteegh, 1989: Nonlinear response to anomalous tropical forcing. J. Atmos. Sci., 46, 3240-3255.

Holton, J. R., 1992: An Introduction to Dynamic Meteorology. Academic Press, 511 pp.

— , and D. E. Colton, 1972: A diagnostic study of the vorticity balance at $200 \mathrm{mb}$ in the tropics during the northern summer. $J$. Atmos. Sci., 29, 1124-1128.

Jin, F.-F., and B. J. Hoskins, 1995: The direct response to tropical heating in a baroclinic atmosphere. J. Atmos. Sci., 52, 307-319.

Kato, T., 1997: The linear response of a global atmosphere to mobile heating. Part 2: Dependency on Rayleigh friction and Newtonian cooling. J. Meteor. Soc. Japan, 75, 867-884.

Lindzen, R. S., 1968: Vertically propagating waves in an atmosphere with Newtonian cooling inversely proportional to density. Can. J. Phys., 46, 1835-1840.

Martinsen, E. A., and J. E. Weber, 1981: Frictional influence on internal Kelvin waves. Tellus, 33, 402-410.

McCreary, J. P., 1981: A linear stratified ocean model of the equatorial undercurrent. Philos. Trans. Roy. Soc. London, A298, 603-635.

Mofjeld, H. O., 1980: Effects of vertical viscosity on Kelvin waves. J. Phys. Oceanogr., 10, 1039-1050.

Newell, R. E., and Z.-X. Wu, 1992: The interrelationship between temperature changes in the free atmosphere and sea surface temperature changes. J. Geophys. Res., 97, 3693-3709.

Plumb, R. A., and J. Eluszkiewicz, 1999: The Brewer-Dobson circulation: Dynamics of the tropical upwelling. J. Atmos. Sci., 56, 868-890.

Rodwell, M. J., and B. J. Hoskins, 1996: Monsoons and the dynamics of deserts. Quart. J. Roy. Meteor. Soc., 122, 1385-1404.

Saravanan, R., and J. C. McWilliams, 1995: Multiple equilibria, natural variability, and climate transitions in an idealized oceanatmosphere model. J. Climate, 8, 2296-2323.

Shapiro, L. J., 1977: Frictional effects on thermally forced waves. Tellus, 29, 264-271.

Wallace, J. M., E. M. Rasmusson, T. P. Mitchell, V. E. Kousky, E. S. Sarachik, and H. von Storch, 1998: On the structure and evolution of ENSO-related climate variability in the tropical Pacific: Lessons from TOGA. J. Geophys. Res., 103, $14241-$ 14259.

Wu, Z., E. S. Sarachik, and D. S. Battisti, 1999: Thermally forced surface winds on an equatorial beta plane. J. Atmos. Sci., 56, 2029-2037.

- D. S. Battisti, and E. S. Sarachik, 2000a: Rayleigh friction, Newtonian cooling, and the linear response to steady tropical heating. J. Atmos. Sci., 57, 1937-1957.

, E. S. Sarachik, and D. S. Battisti, 2000b: Vertical structure of 
convective heating and the three-dimensional structure of the forced circulation in the Tropics. J. Atmos. Sci., 57, 2169-2187. Yamagata, T., and Y. Hayashi, 1984: Oscillation in the tropics. $J$. Meteor. Soc. Japan, 62, 707-717.

, and S. G. H. Philander, 1985: The role of damped equatorial waves in the oceanic response to winds. J. Oceanogr. Soc. Japan, 41, 345-357.

Yulaeva, E., and J. M. Wallace, 1994: The signature of ENSO in global temperature and precipitation fields derived from the microwave sounding unit. J. Climate, 7, 1719-1736. 